Ann. Scient. Éc. Norm. Sup.,

$4^{\text {e }}$ série, t. 39, 2006, p. 983 à 1009.

\title{
FROM TRIANGULATED CATEGORIES TO CLUSTER ALGEBRAS II
}

\author{
By PHILIPPE CALDERO AND BERNHARD KELLER
}

\begin{abstract}
In the acyclic case, we establish a one-to-one correspondence between the tilting objects of the cluster category and the clusters of the associated cluster algebra. This correspondence enables us to solve conjectures on cluster algebras. We prove a multiplicativity theorem, a denominator theorem, and some conjectures on properties of the mutation graph. As in the previous article, the proofs rely on the Calabi-Yau property of the cluster category.
\end{abstract}

(c) 2006 Elsevier Masson SAS

RÉSUMÉ. - Pour le cas des carquois acycliques, nous établissons une correspondance biunivoque entre les objets basculants de la catégorie amassée et les amas de l'algèbre amassée associée. Cette correspondance nous permet de résoudre des conjectures sur les algèbres amassées. Nous prouvons un théorème de multiplication, un théorème de dénominateurs, ainsi que certaines conjectures sur les propriétés du graphe de mutation. Comme dans l'article précédent, les démonstrations reposent sur la propriété de Calabi-Yau de la catégorie amassée.

(c) 2006 Elsevier Masson SAS

\section{Introduction}

Cluster algebras are commutative algebras, introduced in [11] by S. Fomin and A. Zelevinsky. Originally, they were constructed to obtain a better understanding of the positivity and multiplicativity properties of Lusztig's dual (semi)canonical basis of the algebra of coordinate functions on homogeneous spaces. Cluster algebras are generated by the so-called cluster variables gathered into sets of fixed cardinality called clusters. In the framework of the present paper, the cluster variables are obtained by a recursive process from an antisymmetric square matrix $B$.

Denote by $Q$ the quiver associated to the matrix $B$, see 5.1. Assume that $Q$ is connected. A theorem of Fomin and Zelevinsky, [12], asserts that the number of cluster variables of the corresponding cluster algebra $\mathcal{A}_{Q}$ is finite if and only if $Q$ is mutation-equivalent to a quiver whose underlying graph is a simply laced Dynkin diagram. In this case, it is known that the combinatorics of the clusters are governed by the generalized associahedron.

Let $Q$ be any finite quiver without oriented cycles and let $k$ be an algebraically closed field. The cluster category $\mathcal{C}=\mathcal{C}_{Q}$ was introduced in [8] for type $\mathrm{A}_{n}$ and in [6] in the general case. This construction was motivated by the combinatorial similarities of $\mathcal{C}_{Q}$ with the cluster algebra $\mathcal{A}_{Q}$. The cluster category is the category of orbits under an autoequivalence of the bounded derived category $\mathcal{D}^{b}(\bmod k Q)$ of the category of finite dimensional $k Q$-modules. By [18], the category $\mathcal{C}_{Q}$ is a triangulated category. Let us denote its shift functor by $S$ and write $\operatorname{Ext}_{\mathcal{C}}^{1}(M, N)$ for 
$\operatorname{Hom}_{\mathcal{C}}(M, S N)$ for any objects $M, N$ of $\mathcal{C}$. By construction, the cluster category is Calabi-Yau of CY-dimension 2; in other terms, the functor $\mathrm{Ext}^{1}$ is symmetric in the following sense:

$$
\operatorname{Ext}_{\mathcal{C}}^{1}(M, N) \simeq D \operatorname{Ext}_{\mathcal{C}}^{1}(N, M)
$$

In a series of articles [6,3,4], A.B. Buan, R.J. Marsh, I. Reiten and their collaborators study the tilting theory of the cluster category. More precisely, they describe the combinatorics of the cluster tilting objects of the category $\mathcal{C}$, i.e. the objects without self-extensions and with a maximal number of non-isomorphic indecomposable summands. In [4], the authors define a map $\beta$ between the set of clusters of $\mathcal{A}_{Q}$ and the set of tilting objects of the category $\mathcal{C}_{Q}$. A natural question arises: does $\beta$ provide a one-to-one correspondence between both sets?

In Refs. [7,10], it is proved that in the finite case, i.e. the Dynkin case, the cluster algebra can be recovered from the corresponding cluster category as the so-called exceptional Hall algebra of the cluster category. More precisely, in [7] the authors give an explicit correspondence $M \mapsto X_{M}$ between indecomposable objects of $\mathcal{C}_{Q}$ and cluster variables of $\mathcal{A}_{Q}$. In [10], we provide a multiplication rule for the algebra $\mathcal{A}_{Q}$ in terms of the triangulated category $\mathcal{C}_{Q}$.

An ingenious application of the methods of [10] can be found in [14], where the authors give a multiplication formula for elements of Lusztig's dual semicanonical basis. Here, the cluster category is replaced by the category of finite-dimensional modules over the preprojective algebra and the rôle of the cluster algebra is played by the coordinate algebra of the maximal unipotent subgroup in the corresponding semisimple algebraic group.

The aim of the present article is to generalize some of the results of $[7,10]$ to the case where $Q$ is any finite quiver without oriented cycles. Building on the important results obtained in [4] we strengthen here the connections between the cluster category and the cluster algebra by giving an explicit expression for the correspondence $\beta$ and proving that $\beta$ is one-to-one. The key ingredient of the proof is a natural analogue of the map $M \mapsto X_{M}$ of [7]. With the help of a multiplicativity result, we show that $M \mapsto X_{M}$ defines a bijection between the indecomposable objects without self-extensions of $\mathcal{C}_{Q}$ and the cluster variables of $\mathcal{A}_{Q}$.

This correspondence between cluster algebras and cluster categories gives positive answers to some of the conjectures which S. Fomin and A. Zelevinsky formulated in [13]. We prove connectedness properties of some mutation graphs, cf. Section 5.3. As a byproduct, we obtain a cluster-categorical interpretation of the passage to a submatrix of the exchange matrix. This strengthens a key result of [4] and may be of independent interest.

Another consequence of the bijectivity of $\beta$ is that each seed is determined by its cluster. As we have learned recently, this result is obtained independently in [5].

The paper is organized as follows: In the first part, we recall well-known facts on the cluster category. For any object $M$ of the cluster category, we define the Laurent polynomial $X_{M}$ as in [7]. With the techniques of [10], we prove an 'exchange relation' for the $X_{M}$. To be more precise, we prove that if $M$ and $N$ are indecomposable objects of the category $\mathcal{C}=\mathcal{C}_{Q}$ such that $\operatorname{Ext}_{\mathcal{C}}^{1}(M, N)=k$, then

$$
X_{M} X_{N}=X_{B}+X_{B^{\prime}},
$$

where $B$ and $B^{\prime}$ are the unique objects (up to isomorphism) such that there exist non-split triangles

$$
N \rightarrow B \rightarrow M \rightarrow S N, \quad M \rightarrow B^{\prime} \rightarrow N \rightarrow S M .
$$

This formula is an analogue of the 'exchange relation' between cluster variables. With the help of a comparison theorem of [4], we prove by induction that for any indecomposable exceptional object $M, X_{M}$ is a cluster variable and that its (monomial) denominator is given by the

4e SÉRIE - TOME $39-2006-\mathrm{N}^{\circ} 6$ 
dimension vector $\underline{\operatorname{dim}}(M)$. From this denominator property, we deduce that the map $M \mapsto X_{M}$ is injective when restricted to the set of indecomposable objects of $\mathcal{C}_{Q}$ without self-extensions. The connectedness of the tilting graph proved in [4] then implies that the map $M \mapsto X_{M}$ is a one-to-one correspondence between the set of tilting objects of $\mathcal{C}_{Q}$ and the set of clusters of $\mathcal{A}_{Q}$.

We then deduce some applications of this correspondence to conjectures of [13].

\section{The cluster category and the cluster variable formula}

2.1. Let $H$ be a finite dimensional hereditary algebra over an algebraically closed field $k$. We denote by $H$-mod the category of finitely generated $H$-modules. We choose representatives $S_{i}$, $1 \leqslant i \leqslant n$, of the isoclasses of the simple $H$-modules and denote by $I_{i}$ the injective hull and by $P_{i}$ the projective cover of $S_{i}$.

The Grothendieck group of $H$-mod is the group $\mathrm{G}_{0}(H$-mod) generated by the isoclasses of modules in $H$-mod and subject to the relations $X=M+N$ obtained from exact sequences $0 \rightarrow M \rightarrow X \rightarrow N \rightarrow 0$ in $H$-mod. We denote by $[M]$ the class of a module $M$ in $\mathrm{G}_{0}(H$ - $\bmod )$. We put $\alpha_{i}=\left[S_{i}\right]$. The Grothendieck group is free Abelian on the $\alpha_{i}$. The dimension vector $\underline{\operatorname{dim}}(M)$ of a module $M$ is by definition the vector of the coordinates of $[M]$ in this basis.

We define the Euler form by $\langle M, N\rangle=\operatorname{dim} \operatorname{Hom}(M, N)-\operatorname{dim} \operatorname{Ext}^{1}(M, N)$, for any $M, N$ in $H$-mod. Since $H$ is hereditary, this form is well-defined on the Grothendieck group.

Let $\tau$ be the Auslander-Reiten functor of $H$-mod. This functor verifies the Auslander-Reiten formula:

$$
D \operatorname{Hom}(N, \tau M)=\operatorname{Ext}^{1}(M, N),
$$

where $D$ is the functor $\operatorname{Hom}_{k}(?, k)$.

2.2. For any $H$-module $M$, and any $e$ in $\mathrm{G}_{0}\left(H\right.$-mod), we denote by $\operatorname{Gr}_{e}(M)$ the Grassmannian of submodules of $M$ with dimension vector $e$ :

$$
\operatorname{Gr}_{e}(M)=\{N, N \in H-\bmod , N \subset M, \underline{\operatorname{dim}}(N)=e\} .
$$

It is a closed subvariety of the classical Grassmannian of the vector space $M$. Let $\chi_{c}$ be the Euler-Poincaré characteristic of the étale cohomology with $l$-adic coefficients and proper support defined by

$$
\chi_{c}(X)=\sum_{i=0}^{\infty}(-1)^{i} \operatorname{dim} H_{c}^{i}\left(X, \overline{\mathbb{Q}}_{l}\right) .
$$

Let $\mathbb{Q}\left[x_{i}^{ \pm 1}, 1 \leqslant i \leqslant n\right]$ be the $\mathbb{Q}$-algebra of Laurent polynomials in the variables $x_{i}$. As in [7], for any module $M$, we set

$$
X_{M}=\sum_{e} \chi_{c}\left(\operatorname{Gr}_{e}(M)\right) \prod_{i} x_{i}^{-\left\langle e, \alpha_{i}\right\rangle-\left\langle\alpha_{i}, m-e\right\rangle} \in \mathbb{Q}\left[x_{i}^{ \pm 1}, 1 \leqslant i \leqslant n\right],
$$

where $m:=\underline{\operatorname{dim}}(M)$. Note that, as $M$ is finite dimensional, there only exists a finite number of non-zero terms in this sum. Remark that $X_{M}$ only depends on the isoclass of the module $M$. As in [7], one shows that

$$
\chi_{c}\left(\operatorname{Gr}_{g}(M \oplus N)\right)=\sum_{e+f=g} \chi_{c}\left(\operatorname{Gr}_{e}(M)\right) \chi_{c}\left(\operatorname{Gr}_{f}(N)\right) .
$$


Hence, the bilinearity of the Euler form implies that

$$
X_{M \oplus N}=X_{M} X_{N}
$$

2.3. As $H$ is hereditary and finite dimensional, there exists a finite quiver $Q$ without oriented cycles such that $H$ is Morita equivalent to the path algebra $k Q$ of $Q$. Let $Q_{0}$ be the set of vertices and $Q_{1}$ the set arrows of $Q$. Let $n$ be the number of vertices of $Q$.

The bounded derived category $\mathcal{D}^{b}=\mathcal{D}^{b}(H)$ of $H$-mod is a triangulated category. We denote its shift functor $M \mapsto M[1]$ by $S$. The category $\mathcal{D}^{b}$ is a Krull-Schmidt category and, up to canonical triangle equivalence, it only depends on the underlying graph of $Q$, see [15]. We identify the category $H$-mod with the full subcategory of $\mathcal{D}^{b}$ formed by the complexes whose homology is concentrated in degree 0 . We simply call 'modules' the objects in this subcategory. The indecomposable objects of $\mathcal{D}^{b}$ are the shifts $S^{i} M, i \in \mathbb{Z}$, of the indecomposable objects of $H$-mod. We still denote by $\tau$ the AR-functor of $\mathcal{D}^{b}$; it is known that $\tau$ is an autoequivalence characterized by the Auslander-Reiten formula.

Let $F$ be the autoequivalence $\tau^{-1} S$ of $\mathcal{D}^{b}$. The AR-formula implies that

$$
\operatorname{Ext}_{\mathcal{D}^{b}}^{1}(M, N)=\operatorname{Hom}_{\mathcal{D}^{b}}(M, S N)=D \operatorname{Ext}_{\mathcal{D}^{b}}^{1}(F N, M),
$$

for any objects $M, N$ of $\mathcal{D}^{b}$. Let $\mathcal{C}=\mathcal{C}(H)$ be the orbit category $\mathcal{D}^{b} / F$ : the objects of $\mathcal{C}$ are the objects of $\mathcal{D}^{b}$ and the morphisms of $\mathcal{C}$ are given by

$$
\operatorname{Hom}_{\mathcal{C}}(M, N)=\bigoplus_{i \in \mathbb{Z}} \operatorname{Hom}_{\mathcal{D}^{b}}\left(M, F^{i} N\right) .
$$

The category $\mathcal{C}$ is the so-called cluster category, introduced and studied in depth in [6]. Let $\pi$ be the canonical functor from $\mathcal{D}^{b}$ to $\mathcal{C}$. We will often omit the functor $\pi$ from the notations. Statements (i) and (ii) of the following theorem were proved in [18], statements (iii) and (iv) in [6]:

THEOREM 1.-

(i) The category $\mathcal{C}$ is triangulated and

(ii) the functor $\pi: \mathcal{D} \rightarrow \mathcal{C}$ is a triangle functor.

(iii) The category $\mathcal{C}$ is a Krull-Schmidt category and

(iv) for any indecomposable object without self-extensions $M$ of $\mathcal{C}$, we have $\operatorname{End}_{\mathcal{C}}(M)=k$.

The shift functor of the triangulated category $\mathcal{C}$ will still be denoted by $S$. For any objects $M$, $N$ of $\mathcal{C}$, the formulas above imply that there exists an (almost canonical) duality

$$
\phi: \operatorname{Ext}_{\mathcal{C}}^{1}(M, N) \times \operatorname{Ext}_{\mathcal{C}}^{1}(N, M) \rightarrow k
$$

The set of indecomposable objects of $\mathcal{C}$ is given by

$$
\operatorname{lnd}(\mathcal{C})=\operatorname{lnd}(H-\bmod ) \coprod\left\{S P_{i}, 1 \leqslant i \leqslant n\right\}
$$

Note that $S P_{i}=S^{-1} \tau^{-1} S P_{i}=S^{-1} I_{i}$.

We extend the definition of $X_{M}$ to any object $M$ of the category $\mathcal{C}$ by setting $X_{S P_{i}}=x_{i}$, $1 \leqslant i \leqslant n$, and requiring $X_{M \oplus N}=X_{M} X_{N}$ for all objects $M, N$ of $\mathcal{C}$.

$4^{e}$ SÉRIE - TOME $39-2006-\mathrm{N}^{\circ} 6$ 
The AR-formula and the fact that $\tau$ passes to the Grothendieck group of the derived category of $H$-mod allow us to rewrite $X_{M}$ for a module $M$ as

$$
X_{M}=\sum_{e} \chi_{c}\left(\operatorname{Gr}_{e}(M)\right) x^{\tau(e)-\underline{\operatorname{dim}} M+e}
$$

where we have set

$$
x^{v}=\prod_{i=1}^{n} x_{i}^{\left\langle\underline{\operatorname{dim}} S_{i}, v\right\rangle},
$$

for any $v$ in $\mathbb{Z}^{n}$. Remark that this notation gives

$$
X_{S P_{i}}=x \underline{\operatorname{dim}} I_{i} .
$$

2.4. Each object $M$ of $\mathcal{C}$ can be uniquely decomposed in the following way:

$$
M=M_{0} \oplus S P_{M}=M_{0} \oplus S^{-1} I_{M},
$$

where $M_{0}$ is the image under $\pi$ of a module in $\mathcal{D}^{b}$, and where $P_{M}$, respectively $I_{M}$, is a uniquely determined projective, respectively injective, module. We will say that an object $M$ of $\mathcal{C}$ is a module if $M=M_{0}$, and that $M$ is the shift of a projective module if $M=S P_{M}$.

From [6], we recall the

Proposition 1. - For any indecomposable modules $M$ and $N$ in $\mathcal{C}$, we have

$$
\operatorname{Ext}_{\mathcal{C}}^{1}(M, N)=\operatorname{Ext}_{H}^{1}(M, N) \oplus D \operatorname{Ext}_{H}^{1}(N, M) .
$$

The module $M_{0}$ can be recovered using the functor

$$
H^{0}=\operatorname{Hom}_{\mathcal{C}}\left(H_{H}, ?\right): \mathcal{C} \rightarrow H-\bmod .
$$

Indeed, we have

$$
H^{0}(M)=H^{0}\left(M_{0}\right) \oplus H^{0}\left(S P_{M}\right)=\operatorname{Hom}_{H-\bmod }\left(H_{H}, M_{0}\right) \oplus \operatorname{Hom}_{\mathcal{C}}\left(\bigoplus_{i} P_{i}, S P_{M}\right)=M_{0}
$$

as the last factor is zero. The functor $H^{0}$ is a homological functor, i.e. it maps triangles in $\mathcal{C}$ to long exact sequences of $H$-modules.

\section{A multiplication formula}

3.1. The aim of the section is to prove the following theorem:

THEOREM 2.- Let $M$ and $N$ be indecomposable objects of the category $\mathcal{C}$ such that $\operatorname{Ext}_{\mathcal{C}}^{1}(M, N)$ is one-dimensional. Then we have

$$
X_{M} X_{N}=X_{B}+X_{B^{\prime}}
$$

where $B$ and $B^{\prime}$ are the unique objects (up to isomorphism) such that there exist non-split triangles

$$
N \rightarrow B \rightarrow M \rightarrow S N, \quad M \rightarrow B^{\prime} \rightarrow N \rightarrow S M
$$


Note that when $H$ is the path algebra of a Dynkin quiver, the theorem is a particular case of the cluster multiplication formula of [10]. Actually, we will see that the method of [10] generalizes nicely to the framework of the theorem.

Thanks to the hypotheses of the theorem and the symmetry of Ext ${ }^{1}$, we just need to consider the two following cases

(1) $N=S P_{i}$ for an $i \in Q_{0}$ and $M$ is an indecomposable module.

(2) $M$ and $N$ are indecomposable modules.

Indeed, the isomorphisms $M=S P_{j}$ and $N=S P_{i}$ would imply

$$
\operatorname{Ext}_{\mathcal{C}}^{1}(M, N)=\operatorname{Ext}_{\mathcal{C}}^{1}\left(P_{j}, P_{i}\right)=0
$$

3.2. We now prove the theorem in the first case. Suppose $N=S P_{i}$, and let $M$ be an indecomposable module such that $\operatorname{Ext}_{\mathcal{C}}^{1}\left(S P_{i}, M\right)=k$. Using Theorem 1 and the AR-formula, we obtain

$$
\begin{aligned}
\underline{\operatorname{dim}}(M)_{i} & =\operatorname{dim} \operatorname{Hom}_{H}\left(P_{i}, M\right)=\operatorname{dim} \operatorname{Hom}_{\mathcal{C}}\left(P_{i}, M\right) \\
& =\operatorname{dim} \operatorname{Ext}_{\mathcal{C}}^{1}\left(S P_{i}, M\right)=1 .
\end{aligned}
$$

Hence, up to a multiplicative scalar, there exist a unique non-zero morphism $\zeta: M \rightarrow I_{i}$ and a non-zero morphism $\zeta^{\prime}: P_{i} \rightarrow M$.

LEMma 1. - Let $M^{\prime}$ be a submodule of $M$. Then either $M^{\prime} \subset \operatorname{ker} \zeta$ or $\operatorname{im} \zeta^{\prime} \subset M^{\prime}$.

Proof. - By the formula above, the space $M_{i}^{\prime}$ is of dimension 0 or 1 . We claim that

(1) $\operatorname{dim}\left(M^{\prime}\right)_{i}=0$ if and only if $M^{\prime} \subset \operatorname{ker} \zeta$,

(2) $\operatorname{dim}\left(M^{\prime}\right)_{i}=1$ if and only if im $\zeta^{\prime} \subset M^{\prime}$.

The lemma follows from the claim. Let us prove part (1). The second part is similar and left to the reader. The module $\operatorname{im} \zeta$ is non-zero and so it contains the simple socle $S_{i}$ of $I_{i}$. Hence, $\operatorname{dim}(\operatorname{ker} \zeta)_{i}=0$, which gives the 'if' part. Conversely, if $\operatorname{dim}\left(M^{\prime}\right)_{i}=0$, then $\zeta\left(M^{\prime}\right) \cap S_{i}=0$, hence $\zeta\left(M^{\prime}\right)=0$ as $S_{i}$ is the socle of $I_{i}$.

Applying the functor $H^{0}$ to the non-split triangle

$$
S P_{i} \stackrel{\iota}{\longrightarrow} B \stackrel{\pi}{\longrightarrow} M \stackrel{\zeta}{\longrightarrow} S^{2} P_{i}=I_{i}
$$

we obtain a long exact sequence of $H$-modules

$$
0 \longrightarrow H^{0} B \stackrel{H^{0} \pi}{\longrightarrow} M \stackrel{H^{0} \zeta}{\longrightarrow} I_{i} \stackrel{H^{1} \iota}{\longrightarrow} H^{0} \tau B \stackrel{H^{1} \pi}{\longrightarrow} H^{0} \tau M
$$

Now, $H^{0} \tau B=\tau H^{0} B \oplus I_{B}$, and the first factor is non-injective. As the quotient of an injective module is still injective, we have $\operatorname{im}\left(H^{1} \iota\right) \subset I_{B}$. Moreover, as $H^{0} \tau M$ is non-injective, we have $I_{B} \subset \operatorname{ker}\left(H^{1} \pi\right)$. Hence, we have equality and so the following exact sequence holds

$$
0 \longrightarrow H^{0} B \stackrel{H^{0} \pi}{\longrightarrow} M \stackrel{H^{0} \zeta}{\longrightarrow} I_{i} \stackrel{H^{1} \iota}{\longrightarrow} I_{B} \longrightarrow 0 .
$$

Note that the morphism $H^{0} \zeta=\zeta$ is non-zero.

In the same way, applying the functor $H^{0}$ to the non-split triangle

$$
P_{i} \stackrel{\zeta^{\prime}}{\longrightarrow} M \stackrel{\pi^{\prime}}{\longrightarrow} B^{\prime} \stackrel{\iota^{\prime}}{\longrightarrow} S P_{i}
$$

$4^{e}$ SÉRIE - TOME $39-2006-\mathrm{N}^{\circ} 6$ 
we obtain

$$
0 \longrightarrow P_{B^{\prime}} \longrightarrow P_{i} \stackrel{H^{0} \zeta^{\prime}}{\longrightarrow} M \stackrel{H^{0} \pi^{\prime}}{\longrightarrow} H^{0} B^{\prime} \longrightarrow 0 .
$$

Note that the morphism $H^{0} \zeta^{\prime}=\zeta^{\prime}$ is non-zero.

Now, the lemma implies that for any submodule $M^{\prime}$ of $M, M^{\prime}$ either is a submodule of im $H^{0} \pi$ or contains ker $H^{0} \pi^{\prime}$. Hence, there is a natural bijection between $\operatorname{Gr}_{e} M$ and $\mathrm{Gr}_{e}\left(H^{0} B\right) \amalg \mathrm{Gr}_{e-k}\left(H^{0} B^{\prime}\right)$, where

$$
k:=\underline{\operatorname{dim}} \operatorname{ker} H^{0} \pi^{\prime}=\underline{\operatorname{dim}} P_{i}-\underline{\operatorname{dim}} P_{B^{\prime}} .
$$

We want to prove the multiplication formula, which in this case is

$$
\begin{aligned}
x & \stackrel{\operatorname{dim}}{=} I_{i} \sum_{e} \chi_{c}\left(\operatorname{Gr}_{e} M\right) x^{\tau(e)-\underline{\operatorname{dim}} M+e} \\
= & x \underline{\operatorname{dim}} I_{B} \sum_{e} \chi_{c}\left(\operatorname{Gr}_{e} H^{0} B\right) x^{\tau(e)-\underline{\operatorname{dim}}} H^{0} B+e \\
& +x \underline{\operatorname{dim}} I_{B^{\prime}} \sum_{e} \chi_{c}\left(\operatorname{Gr}_{e} H^{0} B^{\prime}\right) x^{\tau(e)-\underline{\operatorname{dim}} H^{0} B^{\prime}+e} .
\end{aligned}
$$

So, it remains to prove that

$$
\underline{\operatorname{dim}} I_{i}+\tau(e)-\underline{\operatorname{dim}} M+e=\underline{\operatorname{dim}} I_{B}+\tau(e)-\underline{\operatorname{dim}} H^{0} B+e,
$$

and

$$
\underline{\operatorname{dim}} I_{i}+\tau(e)-\underline{\operatorname{dim}} M+e=\underline{\operatorname{dim}} I_{B^{\prime}}+\tau(e-k)-\underline{\operatorname{dim}} H^{0} B^{\prime}+e-k .
$$

The first formula is a direct consequence of (3.2). The second one comes from (3.4), (3.5) and the formula $\tau\left(\underline{\operatorname{dim}} P_{j}\right)=-\underline{\operatorname{dim}} I_{j}$.

3.3. This subsection and the following one are devoted to the proof of the theorem in the second case. In order to simplify notations, we will write $(X, Y)$ for $\operatorname{Hom}_{\mathcal{C}}(X, Y)$.

Let $M$ and $N$ be two indecomposable modules such that $\operatorname{Ext}_{\mathcal{C}}^{1}(N, M)=k$. By Proposition 1, we can suppose that $\operatorname{Ext}_{H}^{1}(N, M)=k$ and $\operatorname{Ext}_{H}^{1}(M, N)=0$. In this case, by Theorem 1, there exist (up to isomorphism) a unique non-split short exact sequence of $H$-modules

$$
0 \longrightarrow M \stackrel{i}{\longrightarrow} B_{+} \stackrel{p}{\longrightarrow} N \longrightarrow 0
$$

and two triangles in $\mathcal{C}$

$$
\begin{aligned}
& M \stackrel{i}{\longrightarrow} B_{+} \stackrel{p}{\longrightarrow} N \longrightarrow S M, \\
& N \stackrel{i^{\prime}}{\longrightarrow} B_{-} \stackrel{p^{\prime}}{\longrightarrow} M \longrightarrow S N .
\end{aligned}
$$

Note that $B_{+}$is a 'module' of $\mathcal{C}$ but $B_{-}$is just an object; they both are uniquely determined up to isomorphism. We want to prove the formula

$$
X_{B_{+}}+X_{B_{-}}=X_{M} X_{N}
$$

and the idea is first to construct a morphism $\Psi$ between $\mathrm{Gr} B_{+} \amalg \mathrm{Gr} H^{0} B_{-}$and $\mathrm{Gr} M \times \mathrm{Gr} N$. For any submodule $B_{+}^{\prime}$ of $B_{+}$, set $\Psi\left(B_{+}^{\prime}\right)=\left(i^{-1} B_{+}^{\prime}, p B_{+}^{\prime}\right)$, and for any submodule $B_{-}^{\prime}$ of $B_{-}$, set $\Psi\left(B_{-}^{\prime}\right)=\left(\left(H^{0} p^{\prime}\right) B_{-}^{\prime},\left(H^{0} i^{\prime}\right)^{-1} B_{-}^{\prime}\right)$. As a first step, we want to prove the proposition 
Proposition 2. - The variety $\operatorname{Gr} M \times \operatorname{Gr} N$ is the disjoint union of $\Psi\left(\mathrm{Gr} B_{+}\right)$and $\Psi\left(\mathrm{Gr} H^{0} B_{-}\right)$. Moreover, the fibers of $\Psi$ are affine spaces.

This proposition will be proved at the end of this subsection.

Given a submodule $M^{\prime}$ of $M$, a submodule $N^{\prime}$ of $N$, and the corresponding embeddings $i_{M}$ and $i_{N}$, we have a diagram

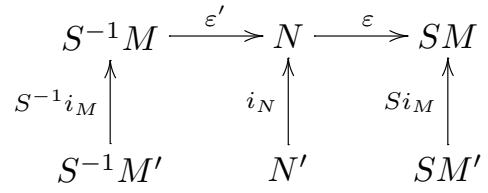

and two complexes

$$
\begin{aligned}
& \left(S^{-1} M, N^{\prime}\right) \stackrel{\alpha^{\prime}}{\longrightarrow}\left(S^{-1} M, N\right) \oplus\left(S^{-1} M^{\prime}, N^{\prime}\right) \stackrel{\beta^{\prime}}{\longrightarrow}\left(S^{-1} M^{\prime}, N\right) \\
& \left(N^{\prime}, S M\right) \underset{\alpha}{\longleftarrow}(N, S M) \oplus\left(N^{\prime}, S M^{\prime}\right) \stackrel{\beta}{\longleftarrow}\left(N, S M^{\prime}\right)
\end{aligned}
$$

where

$$
\begin{aligned}
\alpha^{\prime} & =\left[\begin{array}{c}
\left(i_{N^{\prime}}\right)_{*} \\
\left(S^{-1} i_{M^{\prime}}\right)^{*}
\end{array}\right], \quad \beta^{\prime}=\left[\left(S^{-1} i_{M^{\prime}}\right)^{*},-\left(i_{N}^{\prime}\right)_{*}\right], \\
\alpha & =\left[\left(i_{N^{\prime}}\right)_{*},\left(S i_{M^{\prime}}\right)^{*}\right], \quad \beta=\left[\begin{array}{c}
\left(S i_{M^{\prime}}\right)^{*} \\
-\left(i_{N^{\prime}}\right)_{*}
\end{array}\right] .
\end{aligned}
$$

The two sequences are dual to each other via the canonical duality $\phi$.

The following proposition is straightforward by using basic properties of triangulated categories.

PROPOSITION 3. - The following conditions are equivalent:

(i) There exists a submodule $B_{+}^{\prime} \subset B_{+}$such that the diagram

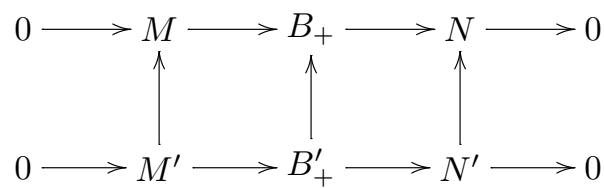

commutes.

(ii) There exists a morphism $\eta: N^{\prime} \rightarrow S M^{\prime}$ such that the square

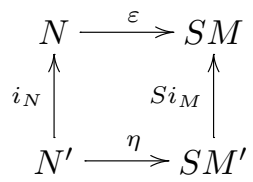

commutes.

(iii) The composed morphism

$$
\operatorname{ker} \alpha \hookrightarrow\left(N^{\prime}, S M^{\prime}\right) \oplus(N, S M) \rightarrow(N, S M)
$$

is non-zero. 
(iv) The composed morphism

$$
\operatorname{cok} \alpha^{\prime} \leftarrow\left(S^{-1} M^{\prime}, N^{\prime}\right) \oplus\left(S^{-1} M, S N\right) \hookleftarrow\left(S^{-1} M, N\right)
$$

is non-zero.

The following proposition sheds light on the situation when the conditions of Proposition 3 do not hold.

PROPOSITION 4. - The following conditions are equivalent:

(i) The composition

$$
\operatorname{cok} \alpha^{\prime} \leftarrow\left(S^{-1} M^{\prime}, N^{\prime \prime}\right) \oplus\left(S^{-1} M, S N\right) \hookleftarrow\left(S^{-1} M, N\right)
$$

vanishes, i.e. $\left(S^{-1} M, N\right)$ is contained in the image of $\alpha^{\prime}$.

(ii) There exist a submodule $B_{-}^{\prime} \hookrightarrow H^{0} B_{-}$and a commutative diagram

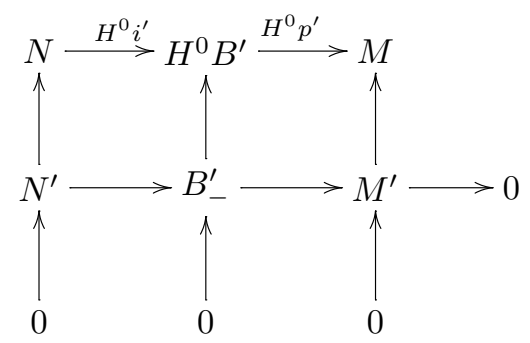

where $M^{\prime}:=H^{0} p^{\prime}\left(B_{-}^{\prime}\right)$ and $N^{\prime}=\left(H^{0} i^{\prime}\right)^{-1}\left(B_{-}^{\prime}\right)$.

Proof. - Let us show that (i) implies (ii). By the assumption, we can find a commutative square

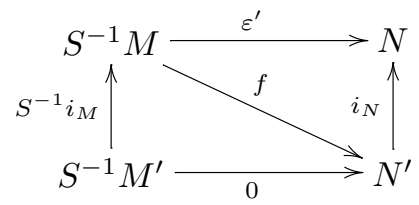

We complete it to a morphism between triangles:

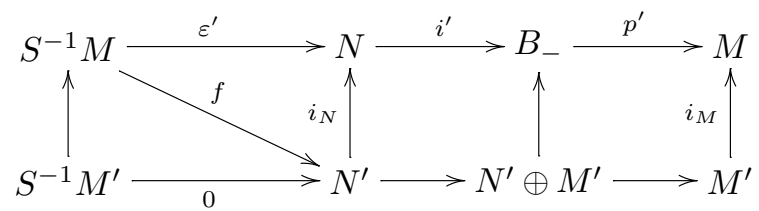

We take the homology:

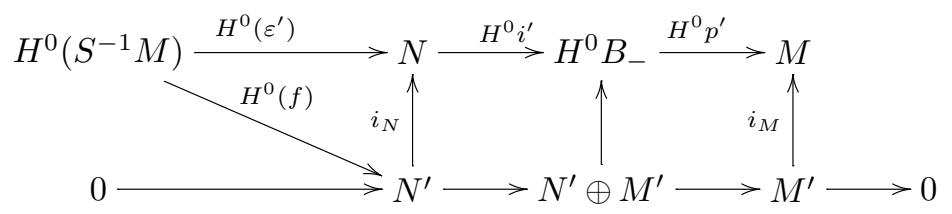

ANNALES SCIENTIFIQUES DE L'ÉCOLE NORMALE SUPÉRIEURE 
We take $B_{-}^{\prime}$ as the image of $N^{\prime} \oplus M^{\prime} \rightarrow H^{0} B_{-}$. Let us show that $N^{\prime} \subset N$ is $H^{0}\left(i^{\prime}\right)^{-1}\left(B_{-}^{\prime}\right)$. Indeed, clearly the image of $N^{\prime}$ is contained in $B_{-}^{\prime}$. Conversely, if we have $x \in N$ whose image lies in $B_{-}^{\prime}$, then the image is the image of $\left(x^{\prime}, y^{\prime}\right)$ in $N^{\prime} \oplus M^{\prime}$, and the image of $x \in N$ under $N \rightarrow H^{0} B_{-} \rightarrow M$ vanishes. So, the image of $y^{\prime}$ in $M$ vanishes. But $M^{\prime} \rightarrow M$ is mono. So $y^{\prime}$ vanishes and we get $x^{\prime}$ in $N^{\prime}$ such that $x$ in $N$ and $x^{\prime}$ have the same image in $H^{0} B_{-}$. Then $x=x^{\prime}+\left(H^{0} \varepsilon^{\prime}\right)(z)$ for some $z$ in $S^{-1} M$. But $H^{0} \varepsilon^{\prime}=\left(H^{0} i_{N}\right) \circ\left(H^{0} f\right)$. So $\left(H^{0} \varepsilon^{\prime}\right)(z)$ lies in fact in $N^{\prime} \subset N$ and $x$ lies in $N^{\prime}$.

Let us show that $M^{\prime}$ is the image of $B_{-}^{\prime}$. Clearly, the image of $B_{-}^{\prime}$ is contained in $M^{\prime}$. Conversely, if $x^{\prime} \in M^{\prime}$, we consider the image $y$ in $B_{-}^{\prime}$ of $\left(0, x^{\prime}\right) \in N^{\prime} \oplus M^{\prime}$. Then clearly, the image of $y$ is $x^{\prime}$.

Let us prove that (ii) implies (i). The hypothesis yields the following diagram

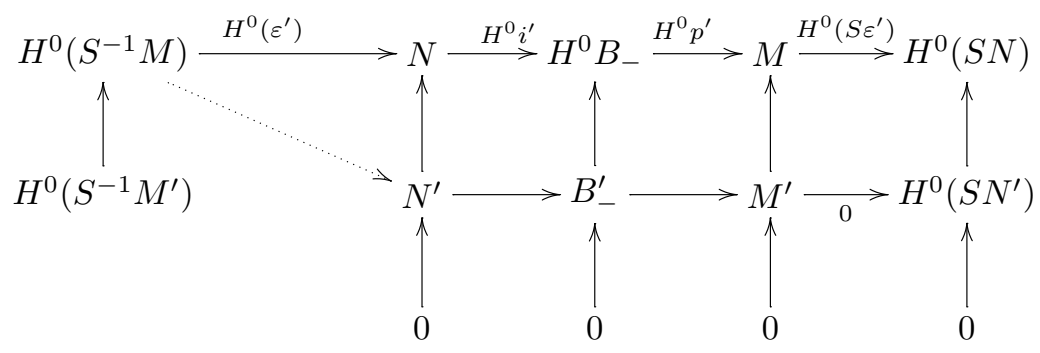

As the composition $H^{0}\left(S^{-1} M\right) \rightarrow N \rightarrow H^{0} B_{-}$vanishes, the image of $H^{0}\left(S^{-1} M\right)$ is contained in $N^{\prime}$, which is the inverse image of $B_{-}^{\prime}$.

As the composition $H^{0}\left(B_{-}\right) \rightarrow M \rightarrow H^{0}(S N)$ vanishes, $M^{\prime}$ is contained in the kernel of $M \rightarrow H^{0}(S N)$. We know that $M$ is not injective, so, $S^{-1} M=\tau^{-1} M$ is still a module.

Moreover, we have

$$
D \operatorname{Ext}^{1}(N, M)=\operatorname{Hom}_{H}\left(\tau^{-1} M, N\right)=\operatorname{Hom}_{H}(M, \tau N)=k .
$$

We obtain the commutative diagrams
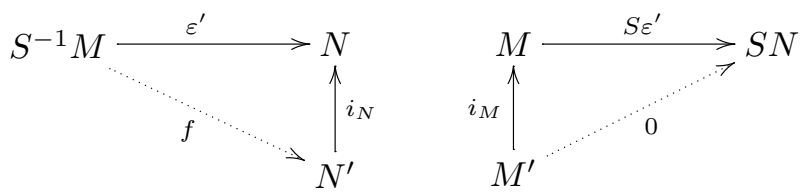

The module $M^{\prime}$ has no injective direct summand, because $M$ is indecomposable and noninjective. So, $S^{-1} M^{\prime}$ is still a module. Consider

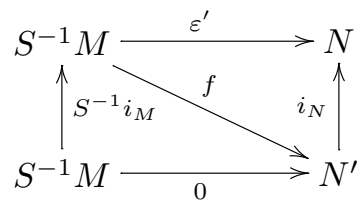

We have $i_{N} \circ f \circ S^{-1} i_{M}=\varepsilon^{\prime} \circ S^{-1} i_{M}=0$. As $i_{N}$ is injective, this gives $f \circ S^{-1} M=0$, which implies (i).

Propositions 3 and 4 imply the first part of Proposition 2. The second part is a well-known fact, cf. Lemma 3.8 of [7].

$4^{\text {e }}$ SÉRIE - TOME $39-2006-\mathrm{N}^{\circ} 6$ 
3.4. We want to prove the multiplication formula for the second case. It reads as follows:

$$
\begin{aligned}
& \sum_{e} \chi_{c}\left(\mathrm{Gr}_{e} M\right) x^{\tau(e)-\underline{\operatorname{dim}} M+e} \sum_{f} \chi_{c}\left(\mathrm{Gr}_{f} N\right) x^{\tau(f)-\underline{\operatorname{dim}} N+f} \\
& =\sum_{g} \chi_{c}\left(\mathrm{Gr}_{g} H^{0} B_{+}\right) x^{\tau(g)-\underline{\operatorname{dim}} B_{+}+g}+x^{\underline{\operatorname{dim}} I_{B_{-}}} \sum_{g} \chi_{c}\left(\mathrm{Gr}_{g} H^{0} B_{-}\right) x^{\tau(g)-\underline{\operatorname{dim}} H^{0} B_{-}+g} .
\end{aligned}
$$

By combining Proposition 2 with Proposition 3.6 of [7], we can compare Euler characteristics on both sides of the equality. What we need to prove now is

$$
\tau(e)-\underline{\operatorname{dim}} M+e+\tau(f)-\underline{\operatorname{dim}} N+f=\tau(g)-\underline{\operatorname{dim}} B_{+}+g,
$$

with $e=\underline{\operatorname{dim}} M^{\prime}, f=\underline{\operatorname{dim}} N^{\prime}, g=\underline{\operatorname{dim}} B_{+}^{\prime}$, in the setting of Proposition 3(i), and then

$$
\tau(e)-\underline{\operatorname{dim}} M+e+\tau(f)-\underline{\operatorname{dim}} N+f=\underline{\operatorname{dim}} I_{B_{-}}+\tau(g)-\underline{\operatorname{dim}} H^{0} B_{-}+g,
$$

with $e=\underline{\operatorname{dim}} M^{\prime}, f=\underline{\operatorname{dim}} N^{\prime}, g=\underline{\operatorname{dim}} B_{-}^{\prime}$, in the setting of Proposition 4(ii).

The formula (3.6) is clear since $g=e+f$ in this case.

In order to prove the second formula, we need to complete the diagram of Proposition 4 by adding kernels and cokernels

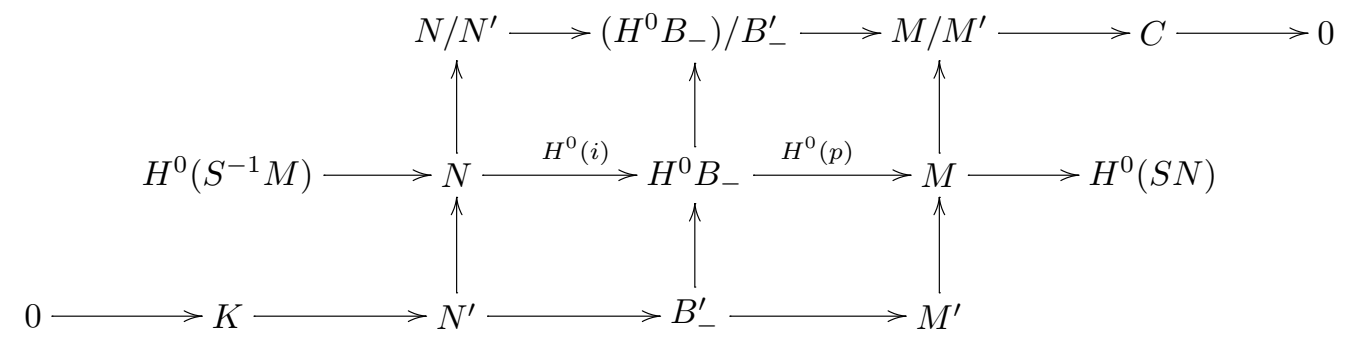

With the notation above, the diagram implies the equalities

$$
\begin{aligned}
& \tau(e)+\tau(f)=\tau(g)+\tau(\underline{\operatorname{dim}} K), \\
& (\underline{\operatorname{dim}} M-e)+(\underline{\operatorname{dim}} N-f)=\left(\underline{\operatorname{dim}} H^{0} B_{-}-g\right)+\underline{\operatorname{dim}} C .
\end{aligned}
$$

So, in order to prove formula (3.7), it remains to show that

$$
\tau \underline{\operatorname{dim}} K-\underline{\operatorname{dim}} C=\underline{\operatorname{dim}} I_{B_{-}} .
$$

For this, we first note that we have the three triangles

$$
\begin{aligned}
& N \stackrel{H^{0}(i)}{\longrightarrow} H^{0} B_{-} \longrightarrow \operatorname{cok}\left(H^{0} i\right) \oplus S K \longrightarrow S N, \\
& S P_{B_{-}} \longrightarrow B_{-} \longrightarrow H^{0} B_{-} \stackrel{0}{\longrightarrow} I_{B_{-}}, \\
& N \longrightarrow Y \longrightarrow S N
\end{aligned}
$$

in $\mathcal{C}_{Q}$. Note that $H^{0} i$ is the composition of the morphism $N \rightarrow B_{-}$with the projection $B_{-} \rightarrow H^{0} B_{-}$. If we form the octahedron associated with this composition, the three triangles we have just mentioned appear among its faces, as well as a new triangle, namely

$$
S P_{B_{-}} \longrightarrow M \longrightarrow \operatorname{cok}\left(H^{0} i\right) \oplus S K \longrightarrow I_{B_{-}} .
$$


If we apply $H^{*}$ to this triangle, we obtain the exact sequence of $H$-modules

$$
0 \longrightarrow M \longrightarrow \operatorname{cok}\left(H^{0} i\right) \oplus H^{0}(\tau K) \longrightarrow I_{B_{-}} \longrightarrow H^{0}(\tau M) .
$$

Since $M$ is an indecomposable module, $\tau M$ is either an indecomposable non-injective module or zero. The image of $I_{B_{-}} \rightarrow \tau M=H^{0} \tau M$ is injective (as a quotient of an injective module). Hence it is zero and we get an exact sequence

$$
0 \longrightarrow M \longrightarrow \operatorname{cok}\left(H^{0} i\right) \oplus H^{0}(\tau K) \longrightarrow I_{B_{-}} \longrightarrow 0 .
$$

In the Grothendieck group, this yields

$$
\begin{aligned}
0 & =\underline{\operatorname{dim}} M-\underline{\operatorname{dim}} \operatorname{cok}\left(H^{0} i\right)-\underline{\operatorname{dim}} H^{0}(\tau K)+\underline{\operatorname{dim}} I_{B_{-}} \\
& =\underline{\operatorname{dim}} C-\underline{\operatorname{dim}} H^{0}(\tau K)+\underline{\operatorname{dim}} I_{B_{-}} .
\end{aligned}
$$

Now, by the third triangle, $K$ is a quotient of $H^{0}\left(S^{-1} M\right)=H^{0}\left(\tau^{-1} M\right)$. As $M$ is a non-injective indecomposable module, $H^{0}\left(\tau^{-1} M\right)=\tau^{-1} M$, so $\tau K$ is a quotient of $M$, and hence, $\tau K$ is a module. Thus, we get formula (3.8) as desired. This ends the proof of Theorem 2.

\section{A denominator theorem}

\subsection{Weakly positive Laurent polynomials}

We recall an idea from [4]: Define an integer polynomial $P$ in $n$ variables $x_{1}, \ldots, x_{n}$ to be weakly positive if we have $P(z)>0$ for each point $z$ of $\mathbb{N}^{n}$ which has at most one vanishing component. If $P$ is an integer polynomial and $d$ an $n$-tuple of integers, then $L=P / x^{d}$ is a Laurent polynomial, where $x^{d}$ is the product of the $x_{i}^{d_{i}}$. It is a weakly positive Laurent polynomial if we can choose $P$ weakly positive. Clearly, in this case, no factor $x_{i}$ divides $P$ so that the factorization $L=\left(1 / x^{d}\right) \cdot P$ is unique. We call $x^{d}$ the denominator of $L$. For example, the Laurent polynomial $L=x_{1}$ is weakly positive with denominator $1 / x_{1}$. The proof of the following lemma is elementary and left to the reader.

LEMMA 2. -

(a) If $L_{1}$ and $L_{2}$ are weakly positive Laurent polynomials, so is their sum $L_{1}+L_{2}$. Moreover, if $x^{d}$ and $x^{e}$ are the denominators of $L_{1}$ and $L_{2}$, the denominator of $L_{1}+L_{2}$ is $x^{\max (d, e)}$, where by definition $\max (d, e)_{i}=\max \left(e_{i}, d_{i}\right), 1 \leqslant i \leqslant n$.

(b) Suppose that $L_{1}$ and $L_{2}$ are Laurent polynomials and $L_{1}$ is weakly positive. Then $L_{2}$ is weakly positive iff $L_{1} L_{2}$ is weakly positive. Moreover, if $x^{d}$ and $x^{e}$ are the denominators of $L_{1}$ and $L_{2}$, the denominator of $L_{1} L_{2}$ is $x^{d+e}$.

\subsection{Denominators and dimension vectors}

From the multiplication formula, we obtain the following denominator property for exceptional modules, i.e. with no self-extensions. A direct proof for arbitrary modules has recently been obtained in [17].

THEOREM 3. - Let $M$ be an indecomposable exceptional $H$-module with dimension vector $\underline{\operatorname{dim}} M=\left(m_{i}\right)$. Then the denominator of $X_{M}$ as an irreducible fraction of integral polynomials in the variables $x_{i}$ is $\prod_{i} x_{i}^{m_{i}}$.

$4^{e}$ SÉRIE - TOME $39-2006-\mathrm{N}^{\circ} 6$ 
Proof. - Let us start with some preliminary remarks: By the explicit formula for $X_{M}$, its denominator as an irreducible fraction of integral polynomials is a monomial

$$
x^{\operatorname{den}(M)}=\prod_{i} x_{i}^{\operatorname{den}(M)_{i}},
$$

where $\operatorname{den}(M) \in \mathbb{Z}^{n}$. We claim that for each exceptional indecomposable $M$, the Laurent polynomial $X_{M}$ is weakly positive. Indeed, if $\left(T, T^{\prime}\right)$ is an exchange pair of exceptional objects (cf. [6]) and

$$
T \rightarrow B \rightarrow T^{\prime} \rightarrow S T \quad \text { and } \quad T^{\prime} \rightarrow B^{\prime} \rightarrow T \rightarrow S T^{\prime}
$$

are non-split triangles, then we have

$$
X_{T^{\prime}}=\frac{X_{B}+X_{B^{\prime}}}{X_{T}}
$$

by the multiplication formula. Thus, by the lemma above, if $X_{B}, X_{B^{\prime}}$ and $X_{T}$ are weakly positive, so is $X_{T^{\prime}}$. The claim therefore follows from the facts that each exceptional object is a direct summand of a cluster tilting object, see (5.2), and that the cluster tilting graph is connected, cf. [6]. The lemma also shows that for an exchange pair $\left(T, T^{\prime}\right)$, we have

$$
\operatorname{den}(T)+\operatorname{den}\left(T^{\prime}\right)=\max \left(\operatorname{den}(B), \operatorname{den}\left(B^{\prime}\right)\right) .
$$

Now for an object $X$ of $\mathcal{C}_{Q}$, we define

$$
\delta(X)=\underline{\operatorname{dim}} H^{0}(X)-\sum m_{i} e_{i},
$$

where $m_{i}$ is the multiplicity of $S P_{i}$ in the decomposition of $X$ into indecomposables and $e_{i}$ the $i$-th vector of the canonical basis of $\mathbb{Z}^{n}$. With this notation, we have to prove that

$$
\delta(M)=\operatorname{den}(M)
$$

for each indecomposable exceptional $M$. If $P$ is indecomposable projective, this equality is trivial for $M=S P$ and is shown for $M=P$ by computing $X_{P}$ explicitly as in [7, Lemma 3.9]. To prove that the equality holds for every indecomposable exceptional, it suffices therefore, by induction, to prove that we have

$$
\delta(T)+\delta\left(T^{\prime}\right)=\max \left(\delta(B), \delta\left(B^{\prime}\right)\right)
$$

if $\left(T, T^{\prime}\right)$ is an exchange pair and $B, B^{\prime}$ are the non-split extensions of $T^{\prime}$ by $T$ and $T$ by $T^{\prime}$, respectively. This will be proved by distinguishing two cases according to whether $T$ and $T^{\prime}$ are modules or one of them is a shifted projective (note that they cannot both be shifted projectives since $\left.\operatorname{Ext}_{\mathcal{C}_{Q}}^{1}\left(T, T^{\prime}\right) \neq 0\right)$.

Case 1: $T$ and $T^{\prime}$ are modules. Since we have

$$
k=\operatorname{Ext}_{\mathcal{C}_{Q}}^{1}\left(T_{1}, T_{1}^{\prime}\right)=\operatorname{Ext}_{k Q}^{1}\left(T_{1}, T_{1}^{\prime}\right) \oplus D \operatorname{Ext}_{k Q}^{1}\left(T_{1}^{\prime}, T_{1}\right),
$$

exactly one of the triangles

$$
T \rightarrow B \rightarrow T^{\prime} \rightarrow S T \quad \text { and } \quad T^{\prime} \rightarrow B^{\prime} \rightarrow T \rightarrow S T^{\prime}
$$


comes from an exact sequence of modules. Let us assume it is the first one. Then, by applying the functor $H^{0}$, we get exact sequences

$$
0 \rightarrow T \rightarrow B \rightarrow T^{\prime} \rightarrow 0 \quad \text { and } \quad T^{\prime} \rightarrow H^{0}\left(B^{\prime}\right) \rightarrow T
$$

These show that we have

$$
\underline{\operatorname{dim}} B=\underline{\operatorname{dim}} T+\underline{\operatorname{dim}} T^{\prime} \text { and } \quad \underline{\operatorname{dim}} H^{0}\left(B^{\prime}\right) \leqslant \underline{\operatorname{dim}} T+\underline{\operatorname{dim}} T^{\prime} \leqslant \underline{\operatorname{dim}} B .
$$

It follows that $\delta\left(B^{\prime}\right) \leqslant \underline{\operatorname{dim}} B=\delta(B)$ and

$$
\delta\left(T^{\prime}\right)+\delta(T)=\delta(B)=\max \left(\delta(B), \delta\left(B^{\prime}\right)\right) .
$$

Case 2: We have $T=S P$ for an indecomposable projective $P$ and $T^{\prime}$ is a module. Again, we have non-split triangles

$$
T \rightarrow B \rightarrow T^{\prime} \rightarrow S T \quad \text { and } \quad T^{\prime} \rightarrow B^{\prime} \rightarrow T \rightarrow S T .
$$

We have

$$
k=\operatorname{Ext}_{\mathcal{C}_{Q}}^{1}\left(T, T^{\prime}\right)=\operatorname{Hom}_{\mathcal{C}_{Q}}\left(S P, S T^{\prime}\right)=\operatorname{Hom}_{k Q}\left(P, T^{\prime}\right)
$$

and

$$
k=\operatorname{Ext}_{\mathcal{C}_{Q}}^{1}\left(T^{\prime}, T\right)=\operatorname{Hom}_{\mathcal{C}_{Q}}\left(T^{\prime}, S^{2} P\right)=\operatorname{Hom}_{k Q}\left(T^{\prime}, \nu P\right),
$$

where $\nu$ is the Nakayama functor. Since $\bmod k Q$ is hereditary, if $f: L \rightarrow M$ is a morphism of $\bmod k Q$, then in the derived category, we have a triangle

$$
L \stackrel{f}{\longrightarrow} M \longrightarrow \operatorname{cok}(f) \oplus S \operatorname{ker}(f) \longrightarrow S L .
$$

It follows that the triangles above are in fact isomorphic to the triangles

$$
\begin{aligned}
& S^{-1} \nu P \rightarrow S^{-1} \operatorname{cok}(f) \oplus \operatorname{ker}(f) \rightarrow T^{\prime} \rightarrow \nu P \quad \text { and } \\
& T^{\prime} \rightarrow S \operatorname{ker}(g) \oplus \operatorname{cok}(g) \rightarrow S P \rightarrow S T^{\prime}
\end{aligned}
$$

associated with arbitrary non-zero morphisms $f: T^{\prime} \rightarrow \nu P$ and $g: P \rightarrow T^{\prime}$. Now $\operatorname{cok}(f)$ is injective as a quotient of an injective and $\operatorname{ker}(g)$ projective as a quotient of a projective. Moreover, if $i$ is the vertex of $Q$ such that $P=P_{i}$, then, as a submodule of $P_{i}$, the module $\operatorname{ker}(f)$ is a direct sum of indecomposables $P_{j}$ such that $j<i$. Similarly, as a quotient of $\nu P_{i}$, the module $\operatorname{cok}(g)$ is a direct sum of indecomposables $\nu P_{j}$ such that $i<j$ (note that we consider right modules and order the vertices of $Q$ in the natural way). It follows that we have

$$
\max \left(\delta\left(S^{-1} \operatorname{cok}(f)\right), \delta(S \operatorname{ker}(g))\right)=0
$$

(note that both vectors have negative components). Thus, we have

$$
\begin{aligned}
\max \left(\delta(B), \delta\left(B^{\prime}\right)\right) & =\max \left(\underline{\operatorname{dim}} \operatorname{ker}(f)+\delta\left(S^{-1} \operatorname{cok}(f)\right), \underline{\operatorname{dim}} \operatorname{cok}(g)+\delta(S \operatorname{ker}(g))\right) \\
& =\max (\underline{\operatorname{dim}} \operatorname{ker}(f), \underline{\operatorname{dim}} \operatorname{cok}(g)) .
\end{aligned}
$$

It remains to be proved that

$$
\underline{\operatorname{dim}} T^{\prime}+\delta(T)=\max (\underline{\operatorname{dim}} \operatorname{ker}(f), \underline{\operatorname{dim}} \operatorname{cok}(g)) .
$$

$4^{\text {e }}$ SÉRIE - TOME $39-2006-\mathrm{N}^{\circ} 6$ 
We check this equality by comparing both sides at each vertex $j$ of $Q$. As above, let $i$ be the vertex of $Q$ such that $P=P_{i}$ so that we have $\delta(T)=-e_{i}$. We have

$$
\operatorname{Hom}\left(P_{i}, T^{\prime}\right)=\operatorname{Ext}_{\mathcal{C}_{Q}}^{1}\left(S P_{i}, T^{\prime}\right)=k
$$

so that $\left(\underline{\operatorname{dim}} T^{\prime}\right)_{i}=1$. The maps $f: T^{\prime} \rightarrow \nu P$ and $g: P \rightarrow T^{\prime}$ induce isomorphisms in $\operatorname{Hom}\left(P_{i}, ?\right)$ since $g \circ f$ induces an isomorphism between the one-dimensional spaces $\operatorname{Hom}\left(P_{i}, P\right)$ to $\operatorname{Hom}\left(P_{i}, \nu P\right)$. It follows that $(\underline{\operatorname{dim}} \operatorname{ker}(f))_{i}$ and $(\underline{\operatorname{dim}} \operatorname{cok}(f))_{i}$ both vanish so that the equality (4.1) holds at $j=i$. Now consider the exact sequences

$$
0 \longrightarrow \operatorname{ker}(f) \longrightarrow T^{\prime} \stackrel{f}{\longrightarrow} \nu P \quad \text { and } \quad P \stackrel{g}{\longrightarrow} T^{\prime} \longrightarrow \operatorname{cok}(g) \longrightarrow 0 .
$$

Suppose that $j$ is not a predecessor of $i$. Then $(\underline{\operatorname{dim}} P)_{j}=0$ and we have $\left(\underline{\operatorname{dim}} T^{\prime}\right)_{j}=$ $(\underline{\operatorname{dim}} \operatorname{cok}(g))_{j}$ by the second sequence and $\left(\underline{\operatorname{dim}} T^{\prime}\right)_{j} \geqslant(\underline{\operatorname{dim}} \operatorname{ker}(f))_{j}$ by the first so that

$$
\left(\underline{\operatorname{dim}} T^{\prime}\right)_{j}=(\underline{\operatorname{dim}} \operatorname{cok}(g))_{j}=\max \left((\underline{\operatorname{dim}} \operatorname{cok}(g))_{j},(\underline{\operatorname{dim}} \operatorname{ker}(g))_{j}\right)
$$

and (4.1) holds at $j$. Similarly, if $j$ is not a successor of $i$, we see that the equality (4.1) holds at $j$. Since $Q$ has no oriented cycles, each vertex $j \neq i$ of $Q$ is a non-successor or a non-predecessor of $i$. Thus, the proof of (4.1) is complete.

4.3. As a corollary of the denominator theorem, we will prove an injectivity property of the map $M \mapsto X_{M}$.

We recall first a few facts on quiver representations.

A representation of $Q$ over a field $F$ is a $Q_{0}$-graded $F$-vector space $V=\bigoplus_{i \in Q_{0}} V_{i}$ together with an element $x=\left(x_{h}\right)_{h \in Q_{1}}$ in $E_{V}:=\prod_{h \in Q_{1}} \operatorname{Hom}\left(V_{s(h)}, V_{t(h)}\right)$, where $s(h)$ is the source and $t(h)$ the target of the arrow $h$. The group $G_{V}:=\prod_{i \in Q_{0}} \mathrm{GL}\left(V_{i}\right)$ acts on $E_{V}$ by $\left(g_{i}\right) \cdot\left(x_{h}\right)=$ $\left(g_{t(h)} x_{h} g_{s(h)}^{-1}\right)$. A representation $(M, x)$ over a field $F$ can be functorialy considered as an $F Q$ module and the dimension vector of this module is $\underline{\operatorname{dim}} M=\left(\operatorname{dim} M_{i}\right)$.

Clearly, the isoclasses of finite-dimensional $F Q$-modules are naturally identified with $G_{V}$-orbits of representations of $Q$.

COROLlARY 1. - If $M$ and $M^{\prime}$ are non-isomorphic indecomposable modules without selfextensions, then $X_{M} \neq X_{M^{\prime}}$.

Proof. - It is well known that in the identification above, an isoclass of $k Q$-module with no self-extension corresponds to an orbit which is dense in its representation space $E_{V}$. Therefore, if $M$ and $M^{\prime}$ are non-isomorphic modules without self-extensions, the corresponding orbits cannot be in the same representation space. Hence, $M$ and $M^{\prime}$ cannot have the same dimension vector.

By the theorem above, we conclude that $X_{M} \neq X_{M^{\prime}}$.

\section{Application to a class of cluster algebras}

5.1. We recall some terminology on cluster algebras. The reader can find more precise and complete information in [12].

Let $n$ be a positive integer. We fix the ambient field $\mathcal{F}=\mathbb{Q}\left(x_{1}, \ldots, x_{n}\right)$, where the $x_{i}$ 's are indeterminates. Let $\mathbf{x}$ be a free generating set of $\mathcal{F}$ over $\mathbb{Q}$ and let $B=\left(b_{i j}\right)$ be an $n \times n$ antisymmetric matrix with coefficients in $\mathbb{Z}$. Such a pair $(\mathbf{x}, B)$ is called $a$ seed. 
Let $(\mathbf{u}, B)$ be a seed and let $u_{j}, 1 \leqslant j \leqslant n$, be in $\mathbf{u}$. We define a new seed as follows. Let $u_{j}^{\prime}$ be the element of $\mathcal{F}$ defined by the exchange relation:

$$
u_{j} u_{j}^{\prime}=\prod_{b_{i j}>0} u_{i}^{b_{i j}}+\prod_{b_{i j}<0} u_{i}^{-b_{i j}} .
$$

Set $\mathbf{u}^{\prime}=\mathbf{u} \cup\left\{u_{j}^{\prime}\right\} \backslash\left\{u_{j}\right\}$. Let $B^{\prime}$ be the $n \times n$ matrix given by

$$
b_{i k}^{\prime}= \begin{cases}-b_{i k} & \text { if } i=j \text { or } k=j \\ b_{i k}+\frac{1}{2}\left(\left|b_{i j}\right| b_{j k}+b_{i j}\left|b_{j k}\right|\right) & \text { otherwise. }\end{cases}
$$

By a result of Fomin and Zelevinsky, $\left(\mathbf{u}^{\prime}, B^{\prime}\right)=\mu_{j}(\mathbf{u}, B)$ is a seed. It is called the mutation of the seed $(\mathbf{u}, B)$ in the direction $u_{j}$ (or $j$ ). We consider all the seeds obtained by iterated mutations. The free generating sets occurring in the seeds are called clusters, and the variables they contain are called cluster variables. By definition, the cluster algebra $\mathcal{A}(\mathbf{x}, B)$ associated to the seed $(\mathbf{x}, B)$ is the $\mathbb{Z}$-subalgebra of $\mathcal{F}$ generated by the set of cluster variables. The graph whose vertices are the seeds and whose edges are the mutations between two seeds is called the mutation graph of the cluster algebra.

The Laurent phenomenon, see [11], asserts that the cluster variables are Laurent polynomials with integer coefficients in the $x_{i}, 1 \leqslant i \leqslant n$. So, we have $\mathcal{A}(\mathbf{x}, B) \subset \mathbb{Z}\left[x_{1}^{ \pm 1}, \ldots, x_{n}^{ \pm 1}\right]$.

Note that an antisymmetric matrix $B$ defines a quiver $Q=Q_{B}$ with vertices corresponding to its rows (or columns) and which has $b_{i j}$ arrows from the vertex $i$ to the vertex $j$ whenever $b_{i j} \geqslant 0$. The quivers $Q$ thus obtained are precisely the finite quivers without oriented cycles of length 1 or 2 . For such quivers $Q$, we denote by $B_{Q}$ the corresponding antisymmetric matrix. The cluster algebra associated to the seed $(\mathbf{x}, B)$ will be also denoted by $\mathcal{A}(Q)$. In the sequel, we will be concerned with cluster algebras associated to a quiver $Q$ without oriented cycles.

5.2. We fix a quiver $Q$ without oriented cycles and we set $H=k Q$. We consider the cluster category $\mathcal{C}=\mathcal{C}_{H}$ associated to the quiver $Q$, cf. [6]. Recall that an object $T$ of $\mathcal{C}$ is called exceptional if it has no self-extensions, i.e. if $\operatorname{Ext}^{1}(T, T)=0$. An exceptional object is called cluster tilting or simply tilting (although this is an abuse of language) if it has $n$ non-isomorphic indecomposable direct summands, where $n$ is the number of vertices of $Q$. In the sequel, we will often identify a tilting object with the datum of its indecomposable summands. An exceptional object is called almost tilting if it has $n-1$ non-isomorphic indecomposable direct summands. It was shown in [6] that any almost tilting object $\bar{T}$ can be completed to precisely two nonisomorphic tilting objects $T$ and $T^{*}$.

For any tilting object $T$ of $\mathcal{C}$, let $Q_{T}$ be the quiver associated to the algebra $\operatorname{End}_{\mathcal{C}}(T)$. To be explicit, fix an ordering of the indecomposable summands $T_{1}, \ldots, T_{n}$ of $T$ and let $A$ be the endomorphism algebra of the sum of the $T_{i}$. Let $e_{i} \in A$ be the idempotent corresponding to $T_{i}$. Then the vertices of $Q_{T}$ are $1, \ldots, n$, and the number of arrows from $i$ to $j$ is equal to $\operatorname{dim} e_{j}\left((\operatorname{rad} A) /(\operatorname{rad} A)^{2}\right) e_{i}$. A pair $\left(T, Q_{T}\right)$ is called a cluster seed.

For $1 \leqslant i \leqslant n$, we define, following [4], the mutation of the cluster seed $\left(T, Q_{T}\right)$ in direction $i$ by

$$
\delta_{i}\left(T, Q_{T}\right):=\left(T^{*}, Q_{T^{*}}\right),
$$

where $T$ and $T^{*}$ are the two completions of the almost tilting object

$$
\bar{T}=T_{1} \oplus \cdots \oplus T_{i-1} \oplus T_{i+1} \oplus \cdots \oplus T_{n} .
$$

$4^{\text {e }}$ SÉRIE - TOME $39-2006-\mathrm{N}^{\circ} 6$ 
Note that there exists an indecomposable object $T_{i}^{*}$, unique up to isomorphism, such that

$$
T^{*}=T_{1} \oplus \cdots \oplus T_{i-1} \oplus T_{i}^{*} \oplus T_{i+1} \oplus \cdots \oplus T_{n}
$$

which provides a natural ordering of the indecomposable summands of $T^{*}$.

The following theorem is the main result of this article. The first assertion is a refinement of Conjecture 9.1 of [6] and the second assertion strengthens the main result of [4].

THEOREM 4. - Let $Q$ be a quiver with $n$ vertices and no oriented cycles, and let $H=k Q$ be the hereditary algebra associated to $Q$. Then

(i) The correspondence $M \mapsto X_{M}$ provides a bijection between the set of indecomposable objects without self-extensions of $\mathcal{C}_{H}$ and the set of cluster variables of $\mathcal{A}(Q)$.

(ii) The correspondence $\left\{T_{1}, \ldots, T_{n}\right\} \mapsto\left\{X_{T_{1}}, \ldots, X_{T_{n}}\right\}$ provides a bijection compatible with mutations between the set of tilting objects of $\mathcal{C}_{H}$ and the set of clusters of $\mathcal{A}(Q)$.

Proof. - By construction, any cluster variable belongs to a cluster. As the map $M \mapsto X_{M}$ is injective on the set of indecomposable objects without self-extensions by Corollary 1, it is enough to prove (ii).

Let us prove (ii). Suppose that $T=T_{1} \oplus \cdots \oplus T_{n}$ is a tilting object of $\mathcal{C}$ and let $T^{*}$ be its mutation in direction $i$. Then $\operatorname{Ext}^{1}\left(T_{i}, T_{i}^{*}\right)$ is one-dimensional by [6]. Hence, by Theorem 2, we have

$$
X_{T_{i}} X_{T_{i}^{*}}=\prod_{j} X_{T_{j}}^{a_{i j}}+\prod_{j} X_{T_{j}}^{c_{i j}},
$$

where $a_{i j}$ and $c_{i j}$ are integers defined by the following non-split triangles (unique up to isomorphism)

$$
\begin{aligned}
& T_{i} \rightarrow \bigoplus a_{i j} T_{j} \rightarrow T_{i}^{*} \rightarrow S T_{i}, \\
& T_{i}^{*} \rightarrow \bigoplus c_{i j} T_{j} \rightarrow T_{i} \rightarrow S T_{i}^{*} .
\end{aligned}
$$

By Theorem 6.2(b) of [4], the quiver $Q_{T}$ is determined by these triangles: for any $i$ and $j$, there are $a_{i j}$ arrows from $i$ to $j$ and $c_{i j}$ arrows from $j$ to $i$. Moreover, if there exists an arrow from $i$ to $j$, then there is no arrow from $j$ to $i$, by Proposition 3.2 of [4].

We now define, as in [4], a correspondence $\beta$ between tilting seeds and cluster seeds. First note that the shift of $H$ is a tilting object and that $(S H, Q)$ is a tilting seed. For a given word $i_{1} \cdots i_{t}$, we can define

$$
\begin{aligned}
& \beta(S H, Q)=\left(\mathbf{x}, B_{Q}\right), \\
& \beta\left(\delta_{i_{t}} \cdots \delta_{i_{1}}(S H, Q)\right)=\mu_{i_{t}} \cdots \mu_{i_{1}}\left(\mathbf{x}, B_{Q}\right) .
\end{aligned}
$$

Set $\left(T, Q_{T}\right):=\delta_{i_{t}} \ldots \delta_{i_{1}}(S H, Q)$. By [4], the quiver obtained from $Q$ by the sequence of tilting mutations in the directions $i_{1}, \ldots, i_{t}$ is equal to the quiver obtained from $Q$ by the sequence of cluster mutations in the directions $i_{1}, \ldots, i_{t}$. Hence, by comparing the cluster exchange relation (5.1) and the tilting exchange relation (5.2), we obtain by induction that

$$
\beta\left(\delta_{i_{t}} \cdots \delta_{i_{1}}(S H, Q)\right)=\left(\left\{X_{T_{1}}, \ldots, X_{T_{n}}\right\}, B_{Q_{T}}\right) .
$$

In particular, $\beta\left(\delta_{i_{t}} \cdots \delta_{i_{1}}(S H, Q)\right)$ does not depend on the choice of the word $i_{1} \cdots i_{t}$.

By Proposition 3.5 of [6], the mutation graph on the set of tilting seeds is connected. Hence, equalities (5.3) and (5.4) define a map $\beta$ from the complete set of tilting seeds to the set of cluster seeds. The surjectivity of $\beta$ follows from the fact that its image is stable under mutation. The injectivity of $\beta$ follows from Corollary 1 . 
5.3. This section is devoted to the proof of some of the conjectures formulated by S. Fomin and A. Zelevinsky in [13]. The first corollary is a straightforward consequence of Theorem 4. It corresponds to [13, Conjecture 4.14(2)] in the acyclic case.

COROLLARY 2. - Let $Q$ be a finite quiver without oriented cycles. Then a cluster seed $(\mathbf{u}, B)$ of $\mathcal{A}(Q)$ only depends on $\mathbf{u}$.

This corollary is [13, Conjecture 4.14(3)] in the acyclic case.

COROLLARY 3. - For any cluster variable $x$, the set of seeds whose clusters contain $x$ forms a connected subgraph of the exchange graph.

Proof. - Indeed, the cluster variable $x$ corresponds to an exceptional indecomposable object $T_{1}$ of $\mathcal{C}_{Q}$. Without restriction of generality, we assume that $T_{1}$ is non-projective. The seeds containing $x$ are in bijection with the completions of $T_{1}$, i.e. the sets $\left\{T_{2}, \ldots, T_{n}\right\}$ of indecomposables such that the sum of the $T_{i}$ is cluster tilting. Two seeds are joined by an edge of the exchange graph iff the corresponding sets of exceptional indecomposables are obtained from each other by a mutation. By [6], this occurs iff they differ by precisely two indecomposables $T_{i}$ and $T_{i}^{*}$ and these satisfy

$$
\operatorname{dim} \operatorname{Ext}^{1}\left(T_{i}, T_{i}^{*}\right)=1 .
$$

This makes it clear that Theorem 5 below yields a bijection compatible with mutations

$$
\left\{T_{2}, \ldots, T_{n}\right\} \mapsto\left\{P T_{2}, \ldots, P T_{n}\right\}
$$

between the completions of $T_{1}$ and the basic tilting sets of $\mathcal{C}_{Q^{\prime}}$, where $Q^{\prime}$ is the quiver of the endomorphism ring of a projective generator of the category $\mathcal{H}^{\prime} \subset \bmod k Q$ of modules $L$ with

$$
\operatorname{Hom}(M, L)=0=\operatorname{Ext}^{1}(M, L) .
$$

Thus, by Theorem 4(ii), the subgraph of the exchange graph of $Q$ formed by the seeds containing $x$ is isomorphic to the exchange graph of $Q^{\prime}$, which is connected by definition.

A consequence of Theorem 4 is also the proof of [13, Conjecture 4.14(4)] in the general case.

COROLlARY 4. - The set of seeds whose matrix is acyclic forms a connected subgraph (possibly empty) of the exchange graph.

Proof. - A seed with an acyclic matrix corresponds to a cluster tilting object $T$ whose endomorphism algebra $A=\operatorname{End}_{\mathcal{C}_{Q}}(T)$ has a quiver without oriented cycles. Thus, the algebra $A$ is both, cluster-tilted and of finite global dimension. By Corollary 2.1 of [19], it is hereditary. So the category $\mathcal{C}_{A}$ is well-defined and the equivalence between the derived categories of $A$ and $Q$ induces a triangle equivalence $\mathcal{C}_{A} \stackrel{\sim}{\longrightarrow} \mathcal{C}_{Q}$ which takes $A$ to $T$. Such an equivalence induces an isomorphism

$$
\Gamma_{A} \rightarrow \Gamma_{B}
$$

of the Auslander-Reiten quivers of the two cluster categories. We refer to [6] for the description of the Auslander-Reiten quivers. Since $A$ is hereditary, the quiver of its indecomposable projectives forms a slice of the component $\Gamma_{A}^{p r}$ of $\Gamma_{A}$ containing the projectives (recall that a slice is a full connected subquiver whose vertices are a system of representatives of the $\tau$-orbits in the component). The isomorphism must take $\Gamma_{A}^{p r}$ to $\Gamma_{B}^{p r}$ since this is the only components isomorphic to the repetition $\mathbb{Z} R$ of a finite quiver $R$. It is clear that any slice of $\Gamma_{B}^{p r}$ can be transformed into the slice of the projectives by finitely many reflections at sources or sinks.

$4^{e}$ SÉRIE - TOME $39-2006-\mathrm{N}^{\circ} 6$ 


\subsection{Cluster tilting objects containing a given summand}

Here, we refine a technique pioneered in Section 2 of [4]: Let $H$ be a finite-dimensional hereditary algebra and $\mathcal{H}$ the category of finite-dimensional right $H$-modules. Let $M \in \mathcal{H}$ be a non-projective indecomposable with $\operatorname{Ext}^{1}(M, M)=0$. Then $\operatorname{End}(M)$ is a (possibly noncommutative) field. Let $\mathcal{H}^{\prime}$ be the full subcategory on the modules $L$ such that

$$
\operatorname{Hom}(M, L)=0 \quad \text { and } \operatorname{Ext}^{1}(M, L)=0 .
$$

We know from $[16,15]$ that $\mathcal{H}^{\prime}$ is a hereditary abelian category with enough projectives and that a projective generator $G$ of $\mathcal{H}^{\prime}$ is obtained by choosing an exact sequence

$$
0 \rightarrow H \rightarrow G \rightarrow M^{r} \rightarrow 0
$$

which induces an isomorphism

$$
\operatorname{Hom}\left(M, M^{r}\right) \stackrel{\sim}{\longrightarrow} \operatorname{Ext}^{1}(M, H)
$$

Let $\mathcal{C}_{\mathcal{H}}$ and $\mathcal{C}_{\mathcal{H}^{\prime}}$ be the cluster categories associated with $\mathcal{H}$ and $\mathcal{H}^{\prime}$. The following theorem is an elaboration on Theorem 2.13 of [4].

THEOREM 5. - Let $\mathcal{C}(\mathcal{H}, M)$ be the full additive subcategory of $\mathcal{C}_{\mathcal{H}}$ whose objects are the sums of indecomposables $L$ of $\mathcal{C}_{\mathcal{H}}$ such that $\operatorname{Ext}^{1}(M, L)=0$. There is a canonical equivalence of $k$-linear categories

$$
P: \mathcal{C}(\mathcal{H}, M) /(M) \stackrel{\sim}{\longrightarrow} \mathcal{C}_{\mathcal{H}^{\prime}},
$$

where $(M)$ denotes the ideal of morphisms factoring through a sum of copies of M. Moreover, we have

$$
\operatorname{Ext}^{1}\left(L_{1}, L_{2}\right) \cong \operatorname{Ext}^{1}\left(P L_{1}, P L_{2}\right)
$$

for all $L_{1}, L_{2} \in \mathcal{C}(\mathcal{H}, M)$.

Note that $\mathcal{C}(\mathcal{H}, M)$ is not a triangulated subcategory and not even stable under the shift functor. The theorem merely claims that as a $k$-linear category, $\mathcal{C}_{\mathcal{H}}$ ' is a 'subquotient' of $\mathcal{C}_{\mathcal{H}}$. To construct the equivalence $P$, we choose a 'fundamental domain' for the action of the autoequivalence $F=\tau^{-1} S$ on $\mathcal{D}$.

Let $\mathcal{P}$ be the full subcategory of the projectives of $\mathcal{H}$ and $\mathcal{H}^{+}$the full additive subcategory of $\mathcal{D}=\mathcal{D}^{b}(\mathcal{H})$ each of whose indecomposables lies in $\mathcal{H}$ or $S \mathcal{P}$. Let $\pi: \mathcal{D} \rightarrow \mathcal{C}_{\mathcal{H}}$ be the projection functor. We know from [6] that $\pi$ induces a bijection from the set of isoclasses of indecomposables of $\mathcal{H}^{+}$to that of $\mathcal{C}_{\mathcal{H}}$ and that we have

$$
\operatorname{Ext}^{1}\left(\pi\left(L_{1}\right), \pi\left(L_{2}\right)\right) \stackrel{\sim}{\longrightarrow} \operatorname{Ext}^{1}\left(L_{1}, L_{2}\right) \oplus D \operatorname{Ext}^{1}\left(L_{2}, L_{1}\right)
$$

for any two indecomposables of $\mathcal{H}^{+}$. Moreover, the category $\mathcal{C}_{\mathcal{H}}$ is equivalent to the category whose objects are those of $\mathcal{H}^{+}$and whose morphisms are given by

$$
\operatorname{Hom}\left(L_{1}, L_{2}\right) \oplus \operatorname{Hom}\left(L_{1}, F L_{2}\right)
$$

with the natural composition. Therefore, Theorem 5 follows from 
THEOREM 6. - There is a canonical bijection $L \mapsto L^{\prime}$ from the set of isoclasses of indecomposables $L$ of $\mathcal{H}^{+}$with

$$
L \neq M, \quad \operatorname{Ext}^{1}(L, M)=0 \quad \text { and } \quad \operatorname{Ext}^{1}(M, L)=0
$$

to the set of isoclasses of indecomposables of $\mathcal{H}^{\prime+}$. Moreover, for any two objects $L_{1}, L_{2}$ of $\mathcal{H}^{+}$ satisfying $(*)$, there is a canonical isomorphism

$$
\operatorname{Ext}^{1}\left(L_{1}, L_{2}\right) \stackrel{\sim}{\longrightarrow} \operatorname{Ext}^{1}\left(L_{1}^{\prime}, L_{2}^{\prime}\right)
$$

and there are canonical isomorphisms

$$
\operatorname{Hom}\left(L_{1}, L_{2}\right) /(M) \stackrel{\sim}{\longrightarrow} \operatorname{Hom}\left(L_{1}^{\prime}, L_{2}^{\prime}\right)
$$

and

$$
\operatorname{Hom}\left(L_{1}, F L_{2}\right) /(M) \stackrel{\sim}{\longrightarrow} \operatorname{Hom}\left(L_{1}^{\prime}, F^{\prime} L_{2}^{\prime}\right)
$$

compatible with compositions.

Before giving the proof, let us illustrate the statement on the following example: We consider the path algebra $H=k Q$ of a linearly oriented quiver $Q$ of type $A_{6}$. Below, we have drawn the Auslander-Reiten quiver of its derived category. The vertices corresponding to indecomposables concentrated in degree 0 lie between the two hatched lines. We use the symbols

$\square$ for the 14 indecomposables $L$ of $\mathcal{H}^{+}$not isomorphic to $M$ and which satisfy

$$
\operatorname{Ext}^{1}(L, M)=0=\operatorname{Ext}^{1}(M, L),
$$

- for the 5 indecomposable projectives of $\mathcal{H}^{\prime}$,

. for indecomposable non-projective objects of $\mathcal{H}^{\prime}$,

$\diamond$ for shifted copies $S P$ of projectives of $\mathcal{H}^{\prime}$.

Notice the two rectangular zones starting from $S^{-1} M$ respectively ending in $S M$ where no occurs. If $L \mapsto L^{\prime}$ denotes the map of the theorem, we have a triangle

$$
L_{\mathcal{U}} \rightarrow L \rightarrow L^{\prime} \rightarrow S L_{\mathcal{U}}
$$

where $L_{\mathcal{U}}$ is a sum of copies of $M$ and $L_{\mathcal{U}} \rightarrow L$ induces a bijection $\operatorname{Hom}\left(M, L_{\mathcal{U}}\right) \stackrel{\sim}{\longrightarrow}$ $\operatorname{Hom}(M, L)$. Thus we have $L=L^{\prime}$ for all $L$ with $\operatorname{Hom}(M, L)=0$. The corresponding triangles for the others are visible in the diagram below:

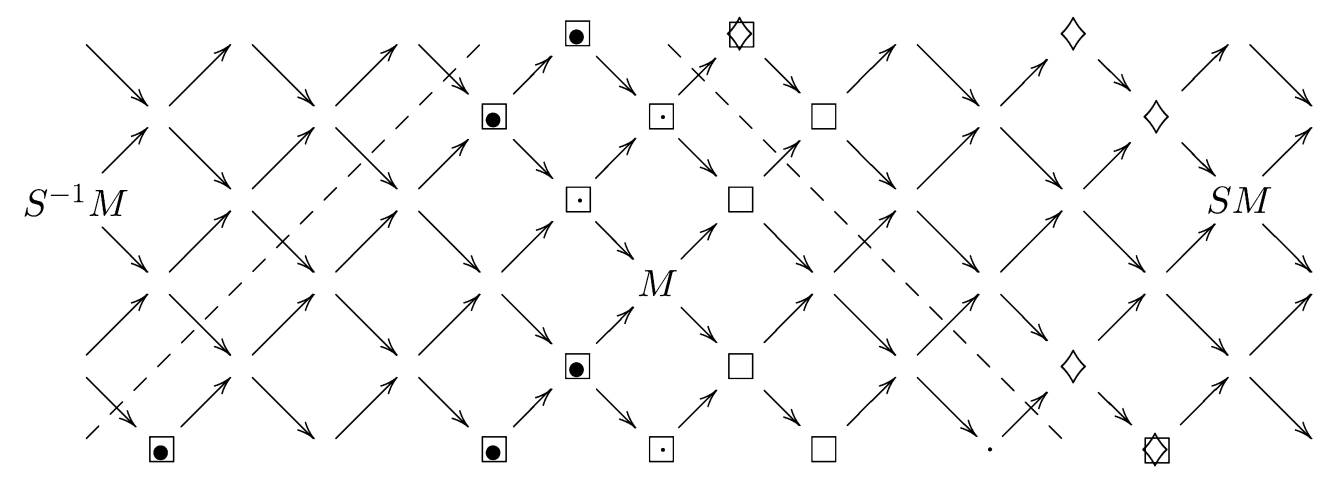

$4^{\text {e }}$ SÉRIE - TOME $39-2006-\mathrm{N}^{\circ} 6$ 
Several of the arguments needed in the proof are contained in Section 2 of [4]. For the convenience of the reader, we nevertheless include them below.

Proof. - Let $\mathcal{U} \subset \mathcal{D}$ be the full triangulated subcategory generated by $M$. Since $\operatorname{Ext}^{1}(M, M)$ vanishes and $\operatorname{Hom}(M, M)$ is a field, its objects are the sums of shifted copies of $M$. Let $\mathcal{V}$ be the full subcategory of $\mathcal{D}$ whose objects are the $L \in \mathcal{D}$ such that $\operatorname{Hom}(U, L)=0$ for all $U \in \mathcal{U}$. Then $\mathcal{U}, \mathcal{V}$ form a semiorthogonal decomposition [1] of $\mathcal{D}$, i.e. for each object $X$ of $\mathcal{D}$, there is a triangle

$$
X_{\mathcal{U}} \rightarrow X \rightarrow X^{\mathcal{V}} \rightarrow S X_{\mathcal{U}}
$$

with $X_{\mathcal{U}} \in \mathcal{U}$ and $X^{\mathcal{V}} \in \mathcal{V}$. This triangle is unique up to unique isomorphism; the functor $X \rightarrow X_{\mathcal{U}}$ is right adjoint to the inclusion of $\mathcal{U}$ and the functor $X \mapsto X^{\mathcal{V}}$ is left adjoint to the inclusion of $\mathcal{V}$. We have $\mathcal{H}^{\prime}=\mathcal{H} \cap \mathcal{V}$ and the inclusion $\mathcal{H}^{\prime} \subset \mathcal{V}$ extends canonically to an equivalence $\mathcal{D}^{b}\left(\mathcal{H}^{\prime}\right) \rightarrow \mathcal{V}$. In particular, each object of $\mathcal{V}$ is a direct sum of shifts of objects of $\mathcal{H}^{\prime}$. We have $\mathcal{U} \cap \mathcal{H}=\mathcal{M}$, the full subcategory on the direct sums of copies of $M$. The inclusion $\mathcal{H}^{\prime} \subset \mathcal{H}$ commutes with kernels, cokernels and preserves Ext ${ }^{1}$-groups. We will show that $L \mapsto L^{\prime}=L^{\mathcal{V}}$ yields the bijection announced in the assertion.

Let $L$ be indecomposable in $\mathcal{H}^{+}$such that $(*)$ holds. Let us first show that $\operatorname{Hom}\left(S^{i} M, L\right)$ vanishes if $i \neq 0$. Indeed, if $L$ belongs to $\mathcal{H}$, then this group clearly vanishes if $i \neq 0,-1$ and if $i=-1$, it vanishes because $\operatorname{Ext}^{1}(M, L)=0$. If $L=S P$ for a projective $P \in \mathcal{H}$, then $\operatorname{Hom}\left(S^{i} M, L\right)=\operatorname{Hom}\left(S^{i} M, S P\right)$ clearly vanishes for $i \neq 0,1$ and it vanishes for $i=1$ because $M$ is a non-projective indecomposable.

Now let us show that $L^{\mathcal{V}}$ is indecomposable: Consider the canonical triangle

$$
L_{\mathcal{U}} \rightarrow L \rightarrow L^{\mathcal{V}} \rightarrow S L_{\mathcal{U}}
$$

Since $\operatorname{Hom}\left(S^{i} M, L\right)$ vanishes for $i \neq 0$, we have $L_{\mathcal{U}} \in \mathcal{M}$. Therefore, in the associated exact sequence

$$
\operatorname{Hom}(L, L) \rightarrow \operatorname{Hom}\left(L, L^{\mathcal{V}}\right) \rightarrow \operatorname{Hom}\left(L, S L_{\mathcal{U}}\right)
$$

the third term vanishes. Thus the composition

$$
\operatorname{Hom}(L, L) \rightarrow \operatorname{Hom}\left(L, L^{\mathcal{V}}\right) \stackrel{\sim}{\longrightarrow} \operatorname{Hom}\left(L^{\mathcal{V}}, L^{\mathcal{V}}\right)
$$

is surjective and $\operatorname{End}\left(L^{\mathcal{V}}\right)$ is local as a quotient of the local ring $\operatorname{End}(L)$.

Let us show that $L^{\prime}$ belongs to $\mathcal{H}^{\prime+}$. Since $L_{\mathcal{U}}$ belongs to $\mathcal{M}$, the canonical morphism $f: L_{\mathcal{U}} \rightarrow L$ is a morphism of $\mathcal{H}$ and therefore its cone $L^{\mathcal{V}}$ in $\mathcal{D}$ is isomorphic to $\operatorname{cok}(f) \oplus$ $S \operatorname{ker}(f)$. Since $L^{\mathcal{V}}$ is indecomposable, one of the two summands vanishes. If $\operatorname{ker}(f)$ vanishes, then $L^{\mathcal{V}}$ belongs to $\mathcal{H}^{\prime} \subset \mathcal{H}^{\prime+}$. If $\operatorname{cok}(f)$ vanishes, we have to show that $\operatorname{ker}(f)$ is projective in $\mathcal{H}^{\prime}$. Now indeed the short exact sequence

$$
0 \rightarrow \operatorname{ker}(f) \rightarrow L_{\mathcal{U}} \rightarrow L \rightarrow 0
$$

induces a surjection

$$
\operatorname{Ext}_{\mathcal{H}}^{1}\left(L_{\mathcal{U}}, U\right) \rightarrow \operatorname{Ext}_{\mathcal{H}}^{1}(\operatorname{ker}(f), U) \rightarrow 0
$$

for each $U \in \mathcal{H}$. The left hand term vanishes since $L_{\mathcal{U}}$ is a sum of copies of $M$ and the right hand term is isomorphic to $\operatorname{Ext}_{\mathcal{H}^{\prime}}^{1}(\operatorname{ker}(f), U)$ because the inclusion $\mathcal{H}^{\prime} \subset \mathcal{H}$ preserves extension groups. Thus, $\operatorname{ker}(f)$ is projective in $\mathcal{H}^{\prime}$. 
From what we have shown, we conclude that the map $L \rightarrow L^{\prime}$ is well-defined. Let us show that it is injective. For this, we show that the morphism $L^{\mathcal{V}} \rightarrow S L_{\mathcal{U}}$ occurring in the canonical triangle is a minimal left $S \mathcal{M}$-approximation. Then $L$ is determined up to isomorphism as the shifted cone over this morphism. To show that $L^{\mathcal{V}} \rightarrow S L_{\mathcal{U}}$ is a minimal left approximation, consider the canonical triangle

$$
L_{\mathcal{U}} \rightarrow L \rightarrow L^{\mathcal{V}} \rightarrow S L_{\mathcal{U}}
$$

and the induced sequence

$$
\operatorname{Hom}(L, S M) \leftarrow \operatorname{Hom}\left(L^{\mathcal{V}}, S M\right) \leftarrow \operatorname{Hom}\left(S L_{\mathcal{U}}, S M\right) .
$$

Since $\operatorname{Hom}(L, S M)$ vanishes by assumption, we do get a surjection $\operatorname{Hom}\left(L^{\mathcal{V}}, S M\right) \leftarrow$ $\operatorname{Hom}\left(S L_{\mathcal{U}}, S M\right)$. If it is not minimal, then there is a retraction $r: S L_{\mathcal{U}} \rightarrow S M$ whose composition with $L^{\mathcal{V}} \rightarrow S L_{\mathcal{U}}$ vanishes. Then $r$ extends to a retraction $\tilde{r}: S L \rightarrow S M$. This is impossible since $L$ is indecomposable and not isomorphic to $M$.

Let us show now that $L \mapsto L^{\prime}$ is surjective. Let $N$ be indecomposable in $\mathcal{H}^{\prime+}$. Let $N \rightarrow S M^{\prime}$ be a minimal $S \mathcal{M}$-approximation and form the triangle

$$
M^{\prime} \rightarrow L \rightarrow N \rightarrow S M^{\prime}
$$

Let us show that $L$ is indecomposable. Since $M^{\prime} \in \mathcal{U}$, we have $L^{\mathcal{V}} \stackrel{\sim}{\longrightarrow} N^{\mathcal{V}}$ and since $N \in \mathcal{V}$, we have $L_{\mathcal{U}} \stackrel{\sim}{\longrightarrow} M^{\prime}$. If $L$ is decomposable, say $L=L_{1} \oplus L_{2}$, then we get

$$
L_{1}^{\mathcal{V}} \oplus L_{2}^{\mathcal{V}} \stackrel{\sim}{\longrightarrow} N
$$

and, say, $L_{1}^{\mathcal{V}}$ vanishes. Then $L_{1}$ belongs to $\mathcal{U}$ and thus $M^{\prime} \stackrel{\sim}{\rightarrow} L_{1} \oplus\left(L_{2}\right)_{\mathcal{U}}$. Since $N \rightarrow S M^{\prime}$ is a minimal $S \mathcal{M}$-approximation, we have $L_{1}=0$. So $L$ is indecomposable.

Let us show that $L$ belongs to $\mathcal{H}^{+}$. It is clear from the above triangle that $L$ has homology at most in degrees 0 and 1 . Since $L$ is indecomposable, its homology is concentrated in one degree. If the homology is concentrated in degree 0 , then $L$ belongs to $\mathcal{H} \subset \mathcal{H}^{+}$. Suppose that $L$ has its homology concentrated in degree 1 . Then we must have $N=S Q$ for some indecomposable projective $Q$ of $\mathcal{H}^{\prime}$. We know that if $P_{H}$ is a projective generator for $\mathcal{H}$, then $P_{H}^{\mathcal{V}}$ is a projective generator for $\mathcal{H}^{\prime}$. Thus, there is a projective $P$ of $\mathcal{H}$ and a section $s: Q \rightarrow P^{\mathcal{U}}$ which identifies $Q$ with a direct factor of $P^{\mathcal{U}}$. Since $N \rightarrow S M^{\prime}$ is an $S \mathcal{M}$-approximation, the composition

$$
N \stackrel{S s}{\longrightarrow} S P^{\mathcal{U}} \longrightarrow S P_{\mathcal{M}}
$$

extends to $S M^{\prime}$ so that we obtain a morphism of triangles

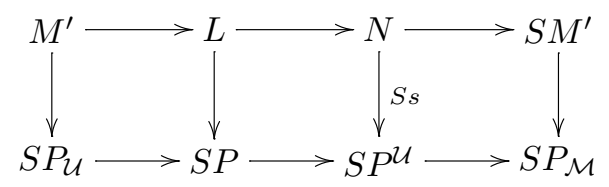

The morphism $L \rightarrow S P$ is non-zero since its composition with $S P \rightarrow S P^{\mathcal{U}}$ equals the composition of the non-zero morphism $L \rightarrow N$ with the section $S s$. So we obtain a non-zero morphism $S^{-1} L \rightarrow P$ in $\mathcal{H}$. Since $S^{-1} L$ is indecomposable and $P$ is projective, $S^{-1} L$ is projective and we have $L \in \mathcal{H}^{+}$.

$4^{\text {e }}$ SÉRIE - TOME $39-2006-\mathrm{N}^{\circ} 6$ 
Finally, let us show that $L$ satisfies the condition $(*)$. If $L$ was isomorphic to $M$, we would have $N=L^{\mathcal{V}}=0$ contrary to our hypothesis that $N$ is indecomposable.

The triangle

$$
M^{\prime} \rightarrow L \rightarrow N \rightarrow S M^{\prime}
$$

yields an exact sequence

$$
\operatorname{Hom}\left(M^{\prime}, S M\right) \leftarrow \operatorname{Hom}(L, S M) \leftarrow \operatorname{Hom}(N, S M) \leftarrow \operatorname{Hom}\left(S M^{\prime}, S M\right) .
$$

Here the leftmost term vanishes since $\operatorname{Ext}^{1}(M, M)=0$ and the rightmost map is surjective since $N \rightarrow S M^{\prime}$ is a left $S \mathcal{M}$-approximation. Thus we have $\operatorname{Ext}^{1}(L, M)=0$. The triangle also yields the sequence

$$
\operatorname{Hom}\left(S^{-1} M, M^{\prime}\right) \rightarrow \operatorname{Hom}\left(S^{-1} M, L\right) \rightarrow \operatorname{Hom}\left(S^{-1} M, N\right)
$$

The left hand term vanishes since $\operatorname{Ext}^{1}\left(L_{1}, M\right)=0$ and the right hand term vanishes since $N$ belongs to $\mathcal{V}$. Thus we have $\operatorname{Ext}^{1}(M, L)=0$.

Now let $L_{1}, L_{2}$ be indecomposables of $\mathcal{H}^{+}$satisfying condition $(*)$. Consider the triangle

$$
\left(L_{2}\right) \mathcal{U} \rightarrow L_{2} \rightarrow L_{2}^{\mathcal{V}} \rightarrow S\left(L_{2}\right) \mathcal{U}
$$

It induces an exact sequence

$$
\begin{aligned}
\operatorname{Hom}\left(S^{-1} L_{1},\left(L_{2}\right) \mathcal{U}\right) & \rightarrow \operatorname{Hom}\left(S^{-1} L_{1}, L_{2}\right) \rightarrow \operatorname{Hom}\left(S^{-1} L_{1}, L_{2}^{\mathcal{V}}\right) \\
& \rightarrow \operatorname{Hom}\left(S^{-1} L_{1}, S\left(L_{2}\right) \mathcal{U}\right) .
\end{aligned}
$$

The leftmost term vanishes since $\operatorname{Ext}^{1}\left(L_{1}, M\right)=0$ and the rightmost term vanishes since $\operatorname{Ext}^{2}\left(L_{1}, M\right)=0$. Thus we have

$$
\operatorname{Hom}\left(L_{1}, S L_{2}\right) \stackrel{\sim}{\longrightarrow} \operatorname{Hom}\left(L_{1}, S L_{2}^{\mathcal{V}}\right) \stackrel{\sim}{\longrightarrow} \operatorname{Hom}\left(L_{1}^{\mathcal{V}}, S L_{2}^{\mathcal{V}}\right)
$$

which proves the assertion on the extension groups. The above triangle also induces an exact sequence

$$
\operatorname{Hom}\left(L_{1},\left(L_{2}\right)_{\mathcal{U}}\right) \rightarrow \operatorname{Hom}\left(L_{1}, L_{2}\right) \rightarrow \operatorname{Hom}\left(L_{1}, L_{2}^{\mathcal{V}}\right) \rightarrow \operatorname{Hom}\left(L_{1}, S\left(L_{2}\right)_{\mathcal{U}}\right)
$$

The last term vanishes since $\operatorname{Ext}^{1}(M, M)=0$. Thus the kernel of the map

$$
\operatorname{Hom}\left(L_{1}, L_{2}\right) \rightarrow \operatorname{Hom}\left(L_{1}, L_{2}^{\mathcal{V}}\right) \stackrel{\sim}{\longrightarrow} \operatorname{Hom}\left(L_{1}^{\mathcal{V}}, L_{2}^{\mathcal{V}}\right)
$$

is formed by the morphisms factoring through sums of $M$. Put $F=\tau^{-1} S$. Consider the triangle

$$
\left(F L_{2}\right)_{\mathcal{U}} \rightarrow F L_{2} \rightarrow\left(F L_{2}\right)^{\mathcal{V}} \rightarrow S\left(F L_{2}\right)_{\mathcal{U}}
$$

Note that the functor $F$ does not take $\mathcal{V}$ to itself. We have

$$
\operatorname{Hom}\left(S^{i} M, F L_{2}\right) \stackrel{\sim}{\longrightarrow} D \operatorname{Hom}\left(L_{2}, S^{i} \tau^{2} M\right) .
$$

This can be non-zero only if $i$ equals 0 or 1 . Thus $\left(F L_{2}\right)_{\mathcal{U}}$ is a sum of copies of $M$ and $S M$. 
Therefore, in the exact sequence

$$
\operatorname{Hom}\left(L_{1},\left(F L_{2}\right) \mathcal{U}\right) \rightarrow \operatorname{Hom}\left(L_{1}, F L_{2}\right) \rightarrow \operatorname{Hom}\left(L_{1},\left(F L_{2}\right)^{\mathcal{V}}\right) \rightarrow \operatorname{Hom}\left(L_{1}, S\left(F L_{2}\right) \mathcal{U}\right)
$$

the last term vanishes and

$$
\operatorname{Hom}\left(L_{1},\left(F L_{2}\right)^{\mathcal{V}}\right) \stackrel{\sim}{\longrightarrow} \operatorname{Hom}\left(L_{1}^{\mathcal{V}},\left(F L_{2}\right)^{\mathcal{V}}\right)
$$

identifies with the quotient of $\operatorname{Hom}\left(L_{1}, F L_{2}\right)$ by the subspace of morphisms factoring through a sum of copies of $M$ and $S M$. Since $\operatorname{Hom}\left(L_{1}, S M\right)$ vanishes, this is also the subspace of morphisms factoring through a sum of copies of $M$. To finish the proof, it remains to be noticed that under the canonical equivalence $\mathcal{D}^{b}\left(\mathcal{H}^{\prime}\right) \stackrel{\sim}{\longrightarrow} \mathcal{V}$, if $L_{2}^{\mathcal{V}}$ corresponds to $L_{2}^{\prime}$, then the object $\left(F L_{2}\right)^{\mathcal{V}}$ does correspond to $\tau_{\mathcal{H}^{\prime}}^{-1} S L_{2}^{\prime}$, by Lemma 2.14 of [4] or Section 8.1 of [18].

\section{Example on the Kronecker quiver}

6.1. We consider the Kronecker quiver $Q$ obtained by choosing the following orientation of the diagram $\tilde{A}_{1}$ :

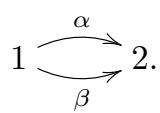

As an illustration of Theorems 2 and 4, we give an interpretation of some results of [20] in terms of the cluster category of the Kronecker quiver. We consider covariant representations. So if $S_{1}$, $S_{2}$ are the simple representations, then

$$
\operatorname{dim} \operatorname{Hom}\left(S_{i}, S_{i}\right)=1, \quad \operatorname{dim} \operatorname{Ext}^{1}\left(S_{1}, S_{2}\right)=2, \quad \operatorname{dim} \operatorname{Ext}^{1}\left(S_{2}, S_{1}\right)=0 .
$$

Over a field $k$, the (finite-dimensional) indecomposable representations of the Kronecker quiver are classified as follows:

The postprojective indecomposable modules $U^{n}, n \geqslant 0$, the preinjective indecomposable modules $V^{n}, n \geqslant 0$, and the family of indecomposable modules $W^{n}=W^{n}(x), n>0$, of the (regular) tube parametrized by $x \in \mathbb{P}^{1}(k)$. They are given by

$$
\begin{aligned}
& U^{n}: k^{n} \underset{\beta}{\stackrel{\alpha}{\longrightarrow}} k^{n+1} \quad \text { with } \alpha=\left[\begin{array}{c}
I_{n} \\
0
\end{array}\right], \beta=\left[\begin{array}{c}
0 \\
I_{n}
\end{array}\right] \text {, } \\
& V^{n}: k^{n+1} \underset{\beta}{\stackrel{\alpha}{\longrightarrow}} k^{n} \quad \text { with } \alpha=\left[\begin{array}{ll}
I_{n} & 0
\end{array}\right], \beta=\left[\begin{array}{ll}
0 & I_{n}
\end{array}\right] \text {, } \\
& W^{n}((1: \lambda)): k^{n} \stackrel{\alpha}{\longrightarrow} k^{n} \quad \text { with } \alpha=I_{n}, \beta=\left(\begin{array}{l}
\lambda \\
0 \\
\vdots \\
\end{array}\right)
\end{aligned}
$$

$4^{\text {e }}$ SÉRIE - TOME $39-2006-\mathrm{N}^{\circ} 6$ 
for $\lambda$ in $k$, and

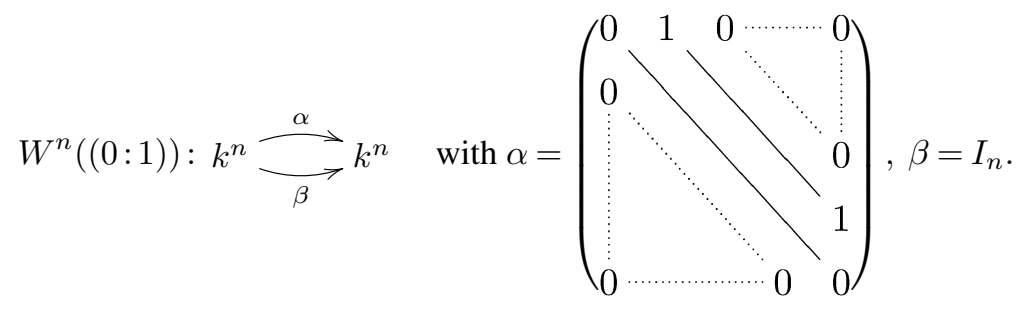

Let us calculate the cluster variables of the cluster algebra $\mathcal{A}(Q) \subset \mathbb{Z}\left[x_{1}^{ \pm 1}, x_{2}^{ \pm 1}\right]$ corresponding to the Kronecker quiver. By Theorem 4, they are given by $X_{U^{n}}, X_{V^{n}}, n \geqslant 0$. By duality, $X_{V^{n}}$ is obtained from $X_{U^{n}}$ by exchanging $x_{1}$ and $x_{2}$. So, we just have to calculate $X_{U^{n}}$.

Let $\left(y_{n}\right)_{n \in \mathbb{N}}$ be the sequence given by $y_{0}=x_{2}, y_{1}=x_{1}$, and $y_{n+2}=X_{U^{n}}, n \geqslant 0$. Set $P_{1}=U^{1}, P_{2}=U^{0}$, for the indecomposable projective modules, then $S P_{2} \oplus S P_{1}$ is the "seed" tilting object and $S P_{1} \oplus U^{0}$ is also a tilting object of the cluster category since the first component of $\underline{\operatorname{dim}}\left(U^{0}\right)$ is zero. Moreover, for any $n \geqslant 0, U^{n} \oplus U^{n+1}$ is easily seen to be a tilting $k Q$-module by applying recursively the inverse AR-functor to the tilting module $P_{2} \oplus P_{1}$ and the object $S P_{1} \oplus U^{0}$. By applying Theorem 4, we obtain that the $y_{n}$ 's are cluster variables and that

$$
\mu_{2}\left(\left\{y_{2 n}, y_{2 n+1}\right\}\right)=\left\{y_{2 n+2}, y_{2 n+1}\right\}, \quad \mu_{1}\left(\left\{y_{2 n-1}, y_{2 n}\right\}\right)=\left\{y_{2 n+1}, y_{2 n}\right\} .
$$

In particular, the exchange relations imply that the sequence $\left(y_{n}\right)_{n \in \mathbb{N}}$ is given by

$$
y_{0}=x_{2}, \quad y_{1}=x_{1}, \quad y_{n-1} y_{n+1}=y_{n}^{2}+1 .
$$

Note that in the module category $\operatorname{Ext}^{1}\left(W^{1}, P_{i}\right)=k$, for $i=1,2$, which implies

$$
\operatorname{Ext}_{\mathcal{C}_{Q}}^{1}\left(W^{1}, P_{i}\right)=k
$$

because the $P_{i}^{\prime} s$ are projective. Applying the (AR)-autoequivalence $\tau$ in the cluster category, we obtain $\operatorname{Ext}_{\mathcal{C}_{Q}}^{1}\left(W^{1}, U^{n}\right)=k, n \geqslant 0$. In the module category, we have (up to isomorphism) a unique non-split exact sequence

$$
0 \rightarrow U^{n} \rightarrow U^{n+1} \rightarrow W^{1} \rightarrow 0 .
$$

This yields a triangle in the cluster category

$$
U^{n} \rightarrow U^{n+1} \rightarrow W^{1} \rightarrow S U^{n}
$$

But, as $S U^{n}=\tau U^{n}=U^{n-2}$ in the cluster category, shifting the triangle gives

$$
W^{1} \rightarrow U^{n-1} \rightarrow U^{n} \rightarrow S W^{1} .
$$

Now, let $w_{1}:=X_{W^{1}}=\frac{1+x_{1}^{2}+x_{2}^{2}}{x_{1} x_{2}}$, then Theorem 2 implies

$$
w_{1} y_{n}=y_{n+1}+y_{n-1}
$$


Note that this formula simplifies the initial induction (6.2). It was obtained in a direct way in [20]. We can calculate the generating series of $\left(y_{n}\right)_{n \in \mathbb{N}}$

$$
\sum_{n \geqslant 0} y_{n} t^{n}=\frac{1-y_{-1} t}{1-w_{1} t+t^{2}}
$$

where $y_{-1}:=X_{V^{0}}=\frac{1+x_{2}^{2}}{x_{1}}$.

\section{Acknowledgements}

The first author is indebted to Thomas Brüstle and Ralf Schiffler for useful conversations. He also wishes to thank Andrei Zelevinsky for his kind hospitality and for pointing out to him the conjectures of [13]. The authors thank Andrew Hubery for pointing out a gap in a previous version of this article.

\section{REFERENCES}

[1] Bondal A.I., KAPRAnov M.M., Representable functors, Serre functors, and reconstructions, Izv. Akad. Nauk SSSR Ser. Mat. 53 (6) (1989) 1183-1205, 1337.

[2] BRENNER S., BUTLER M.C.R., The equivalence of certain functors occurring in the representation theory of Artin algebras and species, J. LMS 14 (1) (1976) 183-187.

[3] Buan A.B., Marsh R.J., Reiten I., Cluster tilted algebras, Trans. Amer. Math. Soc. 359 (2007) 323-332.

[4] Buan A.B., Marsh R.J., Reiten I., Cluster mutation via quiver representations, Comment. Math. Helv., submitted for publication, math.RT/0412077.

[5] Buan A.B., Marsh R.J., Reiten I., Todorov G., Clusters and seeds in acyclic cluster algebras. Appendix by A.B. Buan, R.J. Marsh, P. Caldero, B. Keller, I. Reiten, and G. Todorov, Proc. Amer. Math. Soc., submitted for publication, math.RT/0510359.

[6] Buan A.B., Marsh R.J., Reineke M., Reiten I., Todorov G., Tilting theory and cluster combinatorics, Adv. Math., submitted for publication, math.RT/0402054.

[7] Caldero P., Chapoton F., Cluster algebras as Hall algebras of quiver representations, Comment. Math. Helv. 81 (2006) 595-616, math.RT/0410184.

[8] Caldero P., Chapoton F., SCHIFfler R., Quivers with relations arising from clusters $\left(A_{n}\right.$ case), Trans. Amer. Math. Soc. 358 (2006) 1347-1364.

[9] Caldero P., Chapoton F., Schiffler R., Quivers with relations and cluster tilted algebras, J. Alg. and Rep. Th., submitted for publication, math.RT/0411238.

[10] Caldero P., Keller B., From triangulated categories to cluster algebras, Invent. Math., submitted for publication, math.RT/0506018.

[11] Fomin S., Zelevinsky A., Cluster algebras. I. Foundations, J. Amer. Math. Soc. 15 (2) (2002) 497529.

[12] Fomin S., Zelevinsky A., Cluster algebras. II. Finite type classification, Invent. Math. 154 (1) (2003) 63-121.

[13] Fomin S., ZELEVINSKY A., Cluster algebras: Notes for the CDM-03 conference, math.RT/0311493.

[14] GeISS C., LECLERC B., SCHRÖER J., Rigid modules over preprojective algebras, math.RT/0503324.

[15] Happel D., Triangulated Categories in the Representation Theory of Finite-Dimensional Algebras, London Mathematical Society Lecture Note Series, vol. 119, Cambridge University Press, Cambridge, 1988.

[16] Happel D., Rickard J., Schofield A., Piecewise hereditary algebras, London Math. Soc. 20 (1988) 23-28.

[17] Hubery A., Acyclic cluster algebras via Ringel-Hall algebras, Preprint available at the author's homepage.

$4^{\text {e }}$ SÉRIE - TOME $39-2006-\mathrm{N}^{\circ} 6$ 
[18] Keller B., Triangulated orbit categories, Doc. Math. 10 (2005) 551-581.

[19] Keller B., Reiten I., Cluster tilted algebras are Gorenstein and stably Calabi-Yau, math.RT/0512471.

[20] Sherman P., Zelevinsky A., Positivity and canonical bases in rank 2 cluster algebras of finite and affine types, Mosc. Math. J. 4 (4) (2004) 947-974, 982.

(Manuscrit reçu le 23 avril 2006;

accepté, après révision, le 19 septembre 2006.)

Philippe CALDERO

Institut Camille Jordan,

Université Claude Bernard - Lyon 1,

69622 Villeurbanne cedex, France

E-mail: caldero@math.univ-lyon1.fr

Bernhard KELLER

Université Paris 7 - Denis Diderot,

Institut de Mathématiques,

Case 7012,

2, place Jussieu,

75251 Paris cedex 05, France

E-mail: keller@math.jussieu.fr 